\title{
Bryant surfaces with smooth ends
}

\author{
Christoph Bohle and G. Paul Peters
}

\begin{abstract}
A smooth end of a Bryant surface is a conformally immersed punctured disc of mean curvature 1 in hyperbolic space that extends smoothly through the ideal boundary. The Bryant representation of a smooth end is well defined on the punctured disc and has a pole at the puncture. The Willmore energy of compact Bryant surfaces with smooth ends is quantized. It equals $4 \pi$ times the total pole order of its Bryant representation. The possible Willmore energies of Bryant spheres with smooth ends are $4 \pi\left(\mathbb{N}^{*} \backslash\{2,3,5,7\}\right)$. Bryant spheres with smooth ends are examples of soliton spheres, a class of rational conformal immersions of the sphere which also includes Willmore spheres in the conformal 3-sphere $S^{3}$. We give explicit examples of Bryant spheres with an arbitrary number of smooth ends. We conclude the paper by showing that Bryant's quartic differential $\mathcal{Q}$ vanishes identically for a compact surface in $S^{3}$ if and only if it is the compactification of either a complete finite total curvature Euclidean minimal surface with planar ends or a compact Bryant surface with smooth ends.
\end{abstract}

\section{Introduction}

Surfaces of constant mean curvature 1 in hyperbolic space have attracted much attention since Bryant's fundamental paper [8]. They are nowadays called Bryant surfaces. Bryant surfaces are the hyperbolic analogue to minimal surfaces in Euclidean space, because their Gauss-Mainardi-Codazzi equations are virtually the same. This leads to a local correspondence between Euclidean minimal and Bryant surfaces which is known as the cousin relation. Similar to Euclidean minimal surfaces, which are characterized by the holomorphicity of their Gauss map, Bryant surfaces are characterized by the holomorphicity of their hyperbolic Gauss map. In contrast to the Euclidean minimal case, the hyperbolic Gauss map of a complete finite total curvature Bryant surface may not extend through the ends. The ends at which the hyperbolic Gauss map extends holomorphically are called regular ends, cf. [12, 20,26].

Smooth Bryant ends in hyperbolic space are a direct analog to planar minimal ends in Euclidean space: both extend to immersions through the 
ideal boundary of the space form, i.e., the 2-sphere or the point at infinity, respectively. We show that a Bryant end is smooth if and only if its Bryant representation $F$ is a holomorphic null immersion of the punctured disc into $\mathrm{SL}(2, \mathbb{C})$ with a pole at the puncture such that $F^{\prime} F^{-1}$ has a second-order pole. In other words, a smooth Bryant end is a regular end which is $\mathcal{H}^{3}$ reducible $[21,23]$ (i.e., the representation $F$ has no monodromy) such that $F^{\prime} F^{-1}$ has a second-order pole. The proof is based on a theorem of Collin et al. [12] according to which a properly embedded Bryant annular end is regular. The corresponding characterization in the Euclidean case is that a minimal end is planar if and only if it is the real part of a holomorphic null immersion into $\mathbb{C}^{3}$ with a simple pole at the end. In the case of Bryant surfaces there are two types of smooth ends: those asymptotic to horospheres and those asymptotic to smooth catenoid cousins in the sense of [25]. At a smooth horospherical end $F$ has a simple pole and $F^{\prime} F^{-1}$ automatically has a second-order pole. At a smooth catenoidal end $F$ has a pole of higher order.

Besides the cousin relation there is another correspondence between Bryant surfaces and Euclidean minimal surfaces: if one interprets $\operatorname{SL}(2, \mathbb{C})$ as an affine representation of the complex 3 -quadric $Q^{3} \subset \mathbb{C P}^{4}$ and $\mathbb{C}^{3}$ as stereographic projection of $Q^{3}$ then both types of surfaces are represented by holomorphic null immersions into $Q^{3}$. The $Q^{3}$-valued representation of a smooth Bryant end or a planar Euclidean minimal end extends to an immersion through the end. In case of smooth horospherical Bryant ends and Euclidean minimal planar ends, the holomorphic null immersion transversally intersects the corresponding hyperplane at infinity. In case of smooth catenoidal Bryant ends the intersection is non-transversal.

The analogy goes further if one considers the Willmore energy of surfaces in the conformal 3-sphere $S^{3}=\mathbb{R}^{3} \cup\{\infty\}$ (see Appendix A for the relation of the Willmore energy and the total absolute curvature). As proven by Bryant [7], all complete finite total curvature minimal surfaces with planar ends in $\mathbb{R}^{3}$ extend to compact Willmore surfaces in $S^{3}$, i.e., critical points of the Willmore energy, and all Willmore spheres in $S^{3}$ are obtained this way. Bryant surfaces with smooth ends are not Willmore, but constrained Willmore [6]. Nevertheless, they obey the same quantization of the Willmore energy as Euclidean minimal surfaces with planar ends: in both cases the Willmore energy is $4 \pi d$ where $d \in \mathbb{N}$ is the total pole order of the corresponding null curve in $\mathrm{SL}(2, \mathbb{C})$ or $\mathbb{C}^{3}$, respectively. As in the case of Euclidean minimal spheres with planar ends $[7,9]$, the possible Willmore energies of Bryant spheres with smooth ends are $4 \pi\left(\mathbb{N}^{*} \backslash\{2,3,5,7\}\right)$. In particular, we show that Bryant spheres with exactly $d$ horospherical smooth ends exist if and only if $d \in \mathbb{N}^{*} \backslash\{2,3,5,7\}$. There is no restriction on the number of 


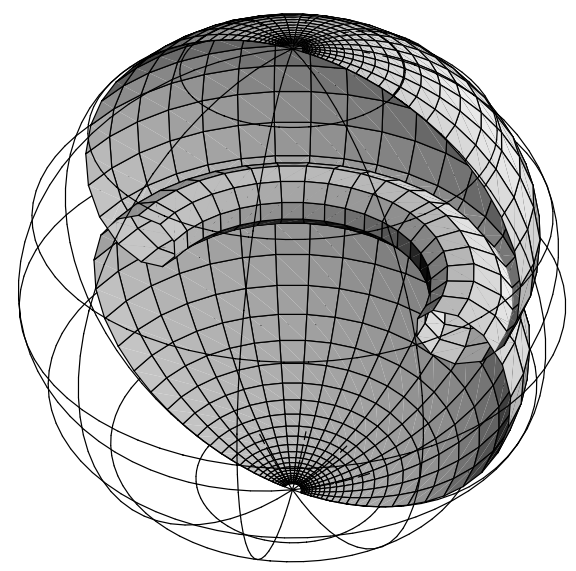

Figure 1: Dirac sphere alias catenoid cousin.

ends if one allows for catenoidal ends: we give explicit rational conformal parametrizations for spheres with an arbitrary number of smooth ends.

We were led to consider Bryant surfaces with smooth ends during our investigations of soliton spheres $[5,18]$ when we observed that the simplest non-trivial Dirac sphere [19], a surface of revolution related to a 1-soliton solution of the mKdV equation [24], is a catenoid cousin with smooth ends, see figure 1. We prove that all Bryant spheres with smooth ends are soliton spheres. The analogous result that all Euclidean minimal spheres with planar ends are soliton spheres is proven in $[5,18]$.

In the last section, we give a uniform Möbius geometric characterization of complete Euclidean minimal surfaces with finite total curvature and planar ends and compact Bryant surfaces with smooth ends in terms of Bryant's quartic differential $\mathcal{Q}$ : we show that $\mathcal{Q}$ vanishes identically for an immersion of a compact surface into $S^{3}$ if and only if it is the compactification of either a Euclidean minimal surface with planar ends or a compact Bryant surface with smooth ends.

\section{The Bryant representation}

The unit 3-ball $\mathbf{B}^{3}=\left\{x \in \mathbb{R}^{3}|| x \mid<1\right\}$ with the metric $d s^{2}=\frac{4|d x|^{2}}{\left(1-|x|^{2}\right)^{2}}$ is the Poincaré ball model of hyperbolic 3 -space. A surface in $\mathbf{B}^{3}$ is called a Bryant surface if it has constant mean curvature one. A fundamental property of Bryant surfaces is that they posses a so-called Bryant representation [8] in terms of holomorphic data similar to the Weierstrass representation of minimal surfaces in $\mathbb{R}^{3}$ : a conformal immersion $f: M \rightarrow \mathbf{B}^{3}$ of a 
Riemann surface $M$ parametrizes a Bryant surface if and only if there exists a holomorphic null immersion $F: \tilde{M} \rightarrow \mathrm{SL}(2, \mathbb{C})$ (null meaning $\operatorname{det} F^{\prime}=0$ ) defined on the universal covering $\tilde{M}$ of $M$ such that

$$
f=\frac{\left(x_{1}, x_{2}, x_{3}\right)}{x_{0}+1}, \quad \text { where }\left(\begin{array}{cc}
x_{0}+x_{3} & x_{1}+x_{2} \mathbf{i} \\
x_{1}-x_{2} \mathbf{i} & x_{0}-x_{3}
\end{array}\right):=F \bar{F}^{t} .
$$

The holomorphic null immersion $F$ representing the Bryant surface $f$ is unique up to right multiplication by a constant $\mathrm{SU}(2)$ matrix. Left multiplication of $F$ with $\mathrm{SL}(2, \mathbb{C})$ matrices yields all congruent Bryant surfaces.

Although the ball model of hyperbolic space is best suited for the definition of smooth ends, the investigation of the ends turns out to be much simpler in the half space model $\mathbf{H}^{3}=\left\{\left(x_{1}, x_{2}, x_{3}\right) \in \mathbb{R}^{3} \mid x_{3}>0\right\}$ with the metric $d s^{2}=\frac{|d x|^{2}}{x_{3}^{2}}$. In the half space model, the Bryant surface corresponding to a holomorphic null immersion $F=\left(\begin{array}{ll}a & b \\ c & d\end{array}\right): \tilde{M} \rightarrow \mathrm{SL}(2, \mathbb{C})$ is given by

$$
x_{1}+\mathbf{i} x_{2}=\frac{a \bar{c}+b \bar{d}}{|c|^{2}+|d|^{2}}, \quad x_{3}=\frac{1}{|c|^{2}+|d|^{2}} .
$$

These formulas may be derived from (2.1) by applying the orientation preserving isometry: $\mathbf{B}^{3} \rightarrow \mathbf{H}^{3},\left(x_{1}, x_{2}, x_{3}\right) \mapsto 2 \frac{\left(x_{1}, x_{2}, 1-x_{3}\right)}{\left|\left(x_{1}, x_{2}, 1-x_{3}\right)\right|^{2}}-(0,0,1)$.

$F$ is a holomorphic null immersion into $\operatorname{SL}(2, \mathbb{C})$ if and only if $F^{-1}$ is a holomorphic null immersion. The surface corresponding to $F^{-1}$ is called the dual Bryant surface of the one represented by $F$.

\section{Smooth ends}

In this section, we characterize Bryant representations of smooth Bryant ends. We reformulate this characterization in terms of holomorphic null immersions into $Q^{3}$. This allows us to determine the possible Willmore energies of Bryant spheres with smooth ends.

Definition. We call a Bryant surface $E$ in the Poincaré ball model $\mathbf{B}^{3} \subset \mathbb{R}^{3}$ of hyperbolic space a smooth Bryant end if there is a point $p_{\infty} \in \partial \mathbf{B}^{3}$ on the asymptotic boundary such that $E \cup\left\{p_{\infty}\right\}$ is a conformally immersed open disc in $\mathbb{R}^{3}$. We call a Bryant surface a compact Bryant surface with smooth ends if it is conformally equivalent to a compact Riemann surface minus a finite number of points such that each of these points has a punctured neighborhood that is a smooth Bryant end. We call it a Bryant sphere with smooth ends if the underlying compact Riemann surface is the sphere. 
The definition is invariant under hyperbolic isometries and one might replace the ball model $\mathbf{B}^{3}$ by the half space model $\mathbf{H}^{3}$ as long as no end goes to $\infty$, because hyperbolic isometries extend to Möbius transformations of the conformal 3-sphere $S^{3}=\mathbb{R}^{3} \cup\{\infty\}$.

We prove now that the Bryant representation of a smooth end is well defined on the punctured unit disc $\Delta^{*}=\Delta \backslash\{0\}$ and has a pole at zero. The proof is based on a result of Collin et al. [12] about the behavior of the holomorphic null immersion $F$ corresponding to a properly embedded Bryant annular end.

Theorem 3.1. If $E$ is a smooth Bryant end, then the corresponding null immersion $F: \tilde{\Delta}^{*} \rightarrow \mathrm{SL}(2, \mathbb{C})$ is well defined on $\Delta^{*}$, has a pole at zero, and $F^{\prime} F^{-1}$ has a pole of order 2 at zero. Conversely, if $F: \Delta^{*} \rightarrow \operatorname{SL}(2, \mathbb{C})$ is a holomorphic null immersion with a pole at zero such that $F^{\prime} F^{-1}$ has a pole of order 2 at zero, then the Bryant surface corresponding to $F$ is a smooth Bryant end.

Remark 3.1. More precisely, Lemma 3.2 implies that if the holomorphic null immersion $F$ has a pole of order 1 at zero, then the Bryant end is asymptotic, in the sense of [25], to the end of a horosphere, and $F^{\prime} F^{-1}$ has automatically a pole of order 2 at zero. If $F$ has a pole of order $n>1$, then the end is asymptotic to the end of a smooth catenoid cousin with Bryant representation

$$
F=\frac{1}{\sqrt{2 \mu+1}}\left(\begin{array}{cc}
(\mu+1) z^{\mu} & \mu z^{-(\mu+1)} \\
\mu z^{\mu+1} & (\mu+1) z^{-\mu}
\end{array}\right)
$$

where $\mu=n-1$, cf. [8]. The fact that the catenoid cousin represented by (3.1) has smooth ends if and only if $\mu=n-1$ can be easily derived from (2.2). A similar observation for trinoids is mentioned in [1].

Proof. Let $E \subset \mathbf{B}^{3}$ be a smooth Bryant end, $f: \Delta \rightarrow \mathbb{R}^{3}$ a conformal immersion such that $f_{\left.\right|_{\Delta^{*}}}$ parametrizes $E$, and $F: \tilde{\Delta}^{*} \rightarrow \mathrm{SL}(2, \mathbb{C})$ the corresponding holomorphic null immersion. Then there is a closed disc $\bar{\Delta}_{\mathrm{r}} \subset \Delta$ such that $f_{\left.\right|_{\bar{\Delta}_{\mathrm{r}}}}$ is a proper embedding into $\mathbf{B}^{3}$. In [12], it is shown in the proofs of Theorems 3 and 4 that $F$ on $\tilde{\Delta}_{\mathrm{r}}^{*}$ is, up to right-multiplication by a constant $\mathrm{SU}(2)$ matrix, the product of a holomorphic $\mathrm{SL}(2, \mathbb{C})$-valued map defined on $\Delta_{\mathrm{r}}^{*}$ with a pole at $z=0$ and $\left(\begin{array}{cc}z^{-\nu} & 0 \\ 0 & z^{\nu}\end{array}\right)$ for some $\nu \in \mathbb{R}$. (One may also derive this, using the description of the behavior of $F$ at regular ends given in [26], from the fact that properly embedded Bryant annular ends are regular [12].) 
Hence there is a holomorphic map $\tilde{F}$ on $\Delta_{\text {r }}$ that does not vanish at zero, $\alpha \in\left[-\frac{1}{2}, \frac{1}{2}\right]$, and $n \in \mathbb{N}$ such that

$$
F(z)=z^{-n} \tilde{F}(z)\left(\begin{array}{cc}
z^{-\alpha} & 0 \\
0 & z^{\alpha}
\end{array}\right)
$$

for all $z \in \Delta_{\mathrm{r}}^{*}$.

We now show that smoothness of $f$ at zero implies $\alpha=0$. Suppose that $\alpha \neq 0$ and let $\tilde{F}=\left(\begin{array}{ll}a & b \\ c & d\end{array}\right)$. Multiplying $F$ by a constant $\operatorname{SL}(2, \mathbb{C})$ matrix from the left and a constant $\mathrm{SU}(2)$ matrix from the right, we may assume that $c(0) \neq 0$ and $a(0)=0$. The conformal immersion into $\mathbf{H}^{3}$ satisfies

$$
x_{1}+\mathbf{i} x_{2}=\frac{a \bar{c}+|z|^{4 \alpha} b \bar{d}}{|c|^{2}+|z|^{4 \alpha}|d|^{2}}, \quad x_{3}=\frac{|z|^{2 n+2 \alpha}}{|c|^{2}+|z|^{4 \alpha}|d|^{2}} .
$$

Thus $x_{1}, x_{2}$ and $x_{3}$ tend to zero if $z$ tends to zero. Therefore, for $f$ to be smooth in particular $x_{3}$ has to be smooth at zero. If $0<\alpha<\frac{1}{2}$, then $x_{3}(z)=o\left(|z|^{2 n}\right)$ but not $O\left(|z|^{2 n+1}\right)$ as $z \rightarrow 0$ and by Taylor's theorem $x_{3}$ could not be $C^{2 n+1}$. Similarly, if $-\frac{1}{2}<\alpha<0$, then $x_{3}(z)=o\left(|z|^{2 n-1}\right)$ but not $O\left(|z|^{2 n}\right)$ as $z \rightarrow 0$ and $x_{3}$ could not be $C^{2 n}$. For $|\alpha|=\frac{1}{2}$ the denominator of $x_{3}$ would be smooth and therefore, because the denominator does not vanish at zero and the numerator $|z|^{2 n \pm 1}$ is not smooth, $x_{3}$ could not be smooth. Thus $\alpha$ has to be zero.

The claim about the pole order of $F^{\prime} F^{-1}$ and the converse follows from Lemma 3.2 below.

We now derive a normal form for the Bryant representation $F$ at a pole.

Lemma 3.1 Normal form. Let $F: \Delta^{*} \rightarrow \mathrm{SL}(2, \mathbb{C})$ be a holomorphic map with a pole of order $n \in \mathbb{N}^{*}$ at zero. Then there exist matrices $A \in \operatorname{SL}(2, \mathbb{C})$, $B \in \mathrm{SU}(2)$, and holomorphic functions $a, b, c, d: \Delta \rightarrow \mathbb{C}$ such that

$$
A F B=z^{-n}\left(\begin{array}{ll}
a & b \\
c & d
\end{array}\right), \quad a(0)=a^{\prime}(0)=b(0)=c(0)=0, \quad d(0) \neq 0 .
$$

If $F$ is null, then the vanishing orders of a and bc at $z=0$ satisfy

$$
\operatorname{ord}_{0}(a) \geq 2 n, \quad \operatorname{ord}_{0}(b c)=2 n \text {. }
$$

In the half plane model (2.2), the fact that $F$ is in normal form implies that the corresponding Bryant end converges to $0 \in \partial \mathbf{H}^{3}$. 
Proof. Since $F$ has a pole of order $n$ at zero there exist holomorphic maps $a, b, c, d: \Delta \rightarrow \mathbb{C}$ such that

$$
F=z^{-n}\left(\begin{array}{ll}
a & b \\
c & d
\end{array}\right)
$$

and one of the functions $a, b, c, d$ does not vanish at zero. Multiplying $F$ by $\left(\begin{array}{cc}0 & -1 \\ 1 & 0\end{array}\right)$ from the left or right we may assume that $d(0) \neq 0$. Multiplying $F$ from the right by

$$
\left(1+\frac{|c(0)|^{2}}{|d(0)|^{2}}\right)^{-\frac{1}{2}}\left(\begin{array}{cc}
1 & \frac{\bar{c}(0)}{\bar{d}(0)} \\
-\frac{c(0)}{d(0)} & 1
\end{array}\right)
$$

one gets $c(0)=0$ while $d(0) \neq 0$ is preserved. Multiplying $F$ from the left by $\left(\begin{array}{cc}1 & -\frac{b(0)}{d(0)} \\ 0 & 1\end{array}\right)$ one obtains $b(0)=0$ while $c(0)=0$ and $d(0) \neq 0$ are preserved. Since $F$ has determinant one (and we did not change this by our multiplications), we have $a d-b c=z^{2 n}$. Thus $a$ vanishes to the second order at zero, because $n \geq 1, d(0) \neq 0$, and $b c$ vanishes to the second order at zero.

If $F$ is null, then $\operatorname{det} F^{\prime}=0$ and $a d-b c=z^{2 n}$ implies $a^{\prime} d^{\prime}-b^{\prime} c^{\prime}=$ $n^{2} z^{2 n-2}$. Suppose $\operatorname{ord}_{0}(b c)=k<2 n$, then $\operatorname{ord}_{0}(a)=k$. Hence $\operatorname{ord}_{0}\left(a^{\prime} d^{\prime}\right) \geq$ $k-1$ and $\operatorname{ord}_{0}\left(b^{\prime} c^{\prime}\right)=k-2<2 n-2$, which is a contradiction to $a^{\prime} d^{\prime}-b^{\prime} c^{\prime}=n^{2} z^{2 n-2}$. If $\operatorname{ord}_{0}(b c)=k>2 n$, then $\operatorname{ord}_{0}(a)=2 n$. Hence $\operatorname{ord}_{0}\left(a^{\prime} d^{\prime}\right) \geq 2 n-1$ and $\operatorname{ord}_{0}\left(b^{\prime} c^{\prime}\right)=k-2>2 n-2$ which again contradicts $a^{\prime} d^{\prime}-b^{\prime} c^{\prime}=n^{2} z^{2 n-2}$. Hence $\operatorname{ord}_{0}(b c)=2 n$ and $\operatorname{ord}_{0}(a) \geq 2 n$.

Lemma 3.2. Let $F: \Delta^{*} \rightarrow \mathrm{SL}(2, \mathbb{C})$ be a holomorphic null immersion with a pole at zero and let $E$ be the Bryant surface corresponding to $F$.

(i) $E$ is a smooth end if and only if $F^{\prime} F^{-1}$ has a pole of order 2 at zero.

(ii) The dual Bryant surface of $E$ is a smooth end if and only if $F^{-1} F^{\prime}$ has a pole of order 2 at zero.

Both $E$ and its dual are smooth ends if and only if $F$ has a pole of order 1.

Proof. Let $n \in \mathbb{N}^{*}$ be the pole order of $F$ and assume that $F$ has the normal form of Lemma 3.1. By (2.2), $E$ has the conformal parametrization

$$
x_{1}+\mathbf{i} x_{2}=\frac{a \bar{c}+b \bar{d}}{|c|^{2}+|d|^{2}}, \quad x_{3}=\frac{|z|^{2 n}}{|c|^{2}+|d|^{2}}
$$

defined on the punctured disc $\Delta^{*}$. The parametrization can be smoothly extended to zero by setting $\left(x_{1}(0), x_{2}(0), x_{3}(0)\right)=0 \in \partial \mathbf{H}^{3}$. 
By Lemma 3.1, $a$ vanishes to the second order at zero and we have

$$
d x_{1}+\mathbf{i} d x_{2}=\frac{b^{\prime} \bar{d}}{|c|^{2}+|d|^{2}} d z+O(|z|) \quad \text { as } z \rightarrow 0 .
$$

Hence $b^{\prime}(0) \neq 0$ if and only if $\left(x_{1}, x_{2}, x_{3}\right): \Delta \rightarrow \mathbb{R}^{3}$ is an immersion, i.e., if and only if $E$ is a smooth end. Because, up to isometry, passing to the dual surface amounts to interchanging $c$ and $b, c^{\prime}(0) \neq 0$ if and only if the dual surface is a smooth end. By Lemma 3.1 we have $\operatorname{ord}_{0}(b c)=2 n$, therefore the case that both $b^{\prime}(0) \neq 0$ and $c^{\prime}(0) \neq 0$ are equivalent to $n=1$.

To complete the proof we have to show that $b^{\prime}(0) \neq 0$ is equivalent to $F^{\prime} F^{-1}$ having a pole of order 2 and $c^{\prime}(0) \neq 0$ is equivalent to $F^{-1} F^{\prime}$ having a pole of order 2 . We have

$$
F^{\prime} F^{-1}=-n z^{-1} \mathrm{Id}+z^{-2 n}\left(\begin{array}{ll}
a^{\prime} d-b^{\prime} c & -a^{\prime} b+b^{\prime} a \\
c^{\prime} d-d^{\prime} c & -c^{\prime} b+d^{\prime} a
\end{array}\right) .
$$

Lemma $3.1 \quad$ implies $\operatorname{ord}_{0}\left(a^{\prime} d-b^{\prime} c\right) \geq 2 n-1, \quad \operatorname{ord}_{0}\left(-a^{\prime} b+b^{\prime} a\right) \geq 2 n$, $\operatorname{ord}_{0}\left(c^{\prime} d-d^{\prime} c\right)=\operatorname{ord}_{0}(c)-1$ and $\operatorname{ord}_{0}\left(-c^{\prime} b+d^{\prime} a\right) \geq 2 n-1$. Hence $F^{\prime} F^{-1}$ has a pole of order 2 if and only if $\operatorname{ord}_{0}(c)=2 n-1$, which is, by Lemma 3.1, equivalent to $b^{\prime}(0) \neq 0$. Because passing to the dual surface essentially interchanges both the roles of $b$ and $c$ and the roles of $F^{\prime} F^{-1}$ and $F^{-1} F^{\prime}$, this also proves the statement for $c^{\prime}(0) \neq 0$.

The following corollary to Theorem 3.1 characterizes the Bryant representations of compact Bryant surfaces with smooth ends.

Corollary 3.1. The Bryant representation F of a compact Bryant surface with smooth ends is a meromorphic null immersion with $\mathrm{SU}(2)$-monodromy of the underlying compact Riemann surface $M$ into $\mathrm{SL}(2, \mathbb{C})$. The poles of $F$ correspond to the ends of the surface. In particular, a Bryant sphere with smooth ends is represented by a rational $\mathrm{SL}(2, \mathbb{C})$-valued null immersion. Conversely, if $F$ is an $\mathrm{SL}(2, \mathbb{C})$-valued null immersion with $S U(2)$ monodromy of a compact Riemann surface $M$ such that $F^{\prime} F^{-1}$ has only poles of order 2, then $F$ represents a compact Bryant surface with smooth ends.

The corollary implies that Bryant spheres with smooth ends are complete $\mathcal{H}^{3}$-reducible Bryant spheres $[21,23]$ of finite total curvature with regular ends, and that all its $\mathcal{H}^{3}$-deformations are Bryant spheres with smooth ends. Examples of the $\mathcal{H}^{3}$-deformation are the warped catenoid cousins [22] represented by $F A$, where $F$ is the representation of a catenoid cousin 

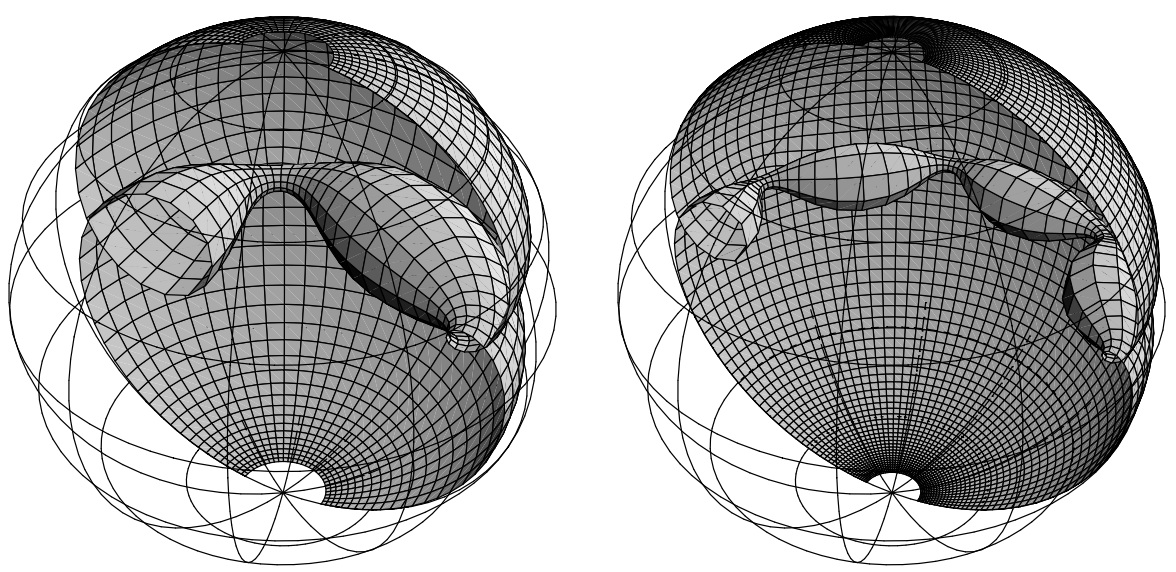

Figure 2: Warped catenoid cousins with smooth ends.

with smooth ends as in $(3.1)$ and $A \in \mathrm{SL}(2, \mathbb{C})$. Figure 2 shows two spheres obtained for $A=\left(\begin{array}{cc}1 & 1 / 2 \\ 0 & 1\end{array}\right)$ and $\mu=1$ or 3 .

A more geometric approach to the study of meromorphic null immersions into $\mathrm{SL}(2, \mathbb{C})$ is to interpret $\mathrm{SL}(2, \mathbb{C})$ as the affine part $\mathbf{Q}^{3} \cap\{e \neq 0\}$ of the quadric

$$
\mathbf{Q}^{3}=\left\{[a, b, c, d, e] \in \mathbb{C P}^{4} \mid a d-b c-e^{2}=0\right\} \subset \mathbb{C P}^{4}
$$

More precisely,

$$
\begin{aligned}
& \mathrm{SL}(2, \mathbb{C}) \stackrel{1: 1}{\longleftrightarrow} \mathrm{Q}^{3} \cap\left\{[a, b, c, d, e] \in \mathbb{C P}^{4} \mid e \neq 0\right\}, \\
& F=\left(\begin{array}{ll}
a & b \\
c & d
\end{array}\right) \longleftrightarrow \Phi=[a, b, c, d, 1] .
\end{aligned}
$$

A holomorphic map $\Phi=[a, b, c, d, e]$ into $\mathbf{Q}^{3}$ is null, i.e., $a^{\prime} d^{\prime}-b^{\prime} c^{\prime}-e^{\prime 2}=0$, if and only if the corresponding $\mathrm{SL}(2, \mathbb{C})$-valued meromorphic map $F=$ $\frac{1}{e}\left(\begin{array}{ll}a & b \\ c & d\end{array}\right)$ is null. By (2.2), the Bryant surface that corresponds to a holomorphic null immersion $\Phi=[a, b, c, d, e]: M \rightarrow \mathbf{Q}^{3}$ is

$$
x_{1}+\mathbf{i} x_{2}=\frac{a \bar{c}+b \bar{d}}{|c|^{2}+|d|^{2}}, \quad x_{3}=\frac{|e|^{2}}{|c|^{2}+|d|^{2}} .
$$

The zeroes of $e$ are the ends of the Bryant surface and the pole order of $F$ at the ends coincides with the intersection order of $\Phi$ with the hyperplane $\{e=0\}$ at the end. This interpretation of meromorphic null maps into $\mathrm{SL}(2, \mathbb{C})$ was suggested to the authors by Ulrich Pinkall. 
The following proposition allows to restate Theorem 3.1 and Corollary 3.1 in terms of $\Phi$.

Proposition 3.1. A holomorphic null map $\Phi: \Delta \rightarrow \mathbf{Q}^{3}$ for which $\Phi(0)$ is contained in the hyperplane $\{e=0\}$ is immersed at 0 if and only if $F^{\prime} F^{-1}$ or $F^{-1} F^{\prime}$ has a pole of order 2 at 0 . The intersection of $\Phi$ with $\{e=0\}$ is transversal if and only if both $F^{\prime} F^{-1}$ and $F^{-1} F^{\prime}$ have poles of order 2 at 0 .

Proof. If the holomorphic map $\Phi: \Delta \rightarrow \mathbf{Q}^{3}$ satisfies $\Phi(0) \in\{e=0\}$ then the corresponding $F: \Delta^{*} \rightarrow \operatorname{SL}(2, \mathbb{C})$ has a pole at zero and there are holomorphic functions $a, b, c, d: \Delta \rightarrow \mathbb{C}$ one of which does not vanish at zero and $n \in \mathbb{N}^{*}$ such that $F=z^{-n}\left(\begin{array}{ll}a & b \\ c & d\end{array}\right)$ and $\Phi=\left[a, b, c, d, z^{n}\right]$. We may assume that $F$ is in normal form of Lemma 3.1, because replacing $F$ by $A F B$ does not change the pole orders of $F^{\prime} F^{-1}$ or $F^{-1} F^{\prime}$ and $\Phi$ changes by a projective transformation that preserves $\{e=0\}$. Then, $a$ vanishes at least to the second order and $d$ does not vanish at zero, so $\Phi$ is immersed if and only if $n=1, b^{\prime}(0) \neq 0$ or $c^{\prime}(0) \neq 0$. As we have seen in the proof of Lemma 3.2, this is equivalent to $F^{\prime} F^{-1}$ or $F^{-1} F^{\prime}$ having a pole of order 2 at zero.

Theorem 3.1 and Corollary 3.1 can now be restated as follows:

Theorem 3.1'. If $E$ is a smooth Bryant end, then the corresponding null immersion $\Phi: \tilde{\Delta}^{*} \rightarrow \mathbf{Q}^{3}$ is well defined on $\Delta^{*}$ and extends to a holomorphic immersion on $\Delta$. Conversely, if $\Phi: \Delta \rightarrow \mathrm{Q}^{3}$ is a holomorphic null immersion such that $\Phi(0)$ is contained in the hyperplane $\{e=0\}$, then the corresponding Bryant surface or its dual Bryant surface is a smooth Bryant end.

The generic case, i.e., $\Phi$ intersects the hyperplane $\{e=0\}$ transversally, is equivalent to both surfaces being smooth horospherical Bryant ends.

Corollary 3.1'. A compact Bryant surface with smooth ends is represented by a holomorphic null immersion $\Phi$ of the universal covering $\tilde{M}$ of a compact Riemann surface $M$ into $\mathbf{Q}^{3}$. In particular, a Bryant sphere with smooth ends is represented by a rational null immersion into $\mathbf{Q}^{3}$.

The fact that Bryant spheres with smooth ends are represented by rational null immersions into $\mathbf{Q}^{3}$ provides a strong link to Willmore spheres in $S^{3}$, because these as well are related to rational null immersion into $\mathbf{Q}^{3}$, see [7]. As in the case of Willmore spheres, the Willmore energy of a Bryant sphere with smooth ends is given by $4 \pi \operatorname{deg} \Phi$. This follows from Theorem 5.1 
below, because $\operatorname{deg} \Phi$ is the total pole order of $F$. Using Bryant's result [9] that the possible degrees $d$ of rational null immersions into $\mathbf{Q}^{3}$ are the numbers $d \in \mathbb{N}^{*} \backslash\{2,3,5,7\}$, we obtain the following theorem.

Theorem 3.2. The possible Willmore energies of Bryant spheres with smooth ends are $4 \pi d$ with $d \in \mathbb{N}^{*} \backslash\{2,3,5,7\}$.

Corollary 3.2. Bryant spheres with $d$ smooth ends that are all horospherical exist if and only if $d \in \mathbb{N}^{*} \backslash\{2,3,5,7\}$.

The generic case of horospherical smooth ends does not occur among the rotationally symmetric examples of the catenoid cousins. However, generic projective transformations of $\mathbb{C P}^{4}$ that preserve $\mathrm{Q}^{3}$ deform catenoidal smooth ends into horospherical smooth ends. As an example, we apply the projective transformation $a \mapsto a, d \mapsto d,\left(\begin{array}{cc}e & b \\ c & -e\end{array}\right) \mapsto A^{-1}\left(\begin{array}{ll}e & b \\ c & -e\end{array}\right) A, A=$ $\left(\begin{array}{cc}1 & 0 \\ -t & 1\end{array}\right)\left(\begin{array}{ll}1 & s \\ 0 & 1\end{array}\right)$ to the holomorphic null immersion $\Phi=[a, b, c, d, e]$ of a catenoid cousin. If $\mu=n-1, n \in \mathbb{N}^{*} \backslash\{1\}$ in $(3.1)$ and $s t \in \mathbb{C}^{*} \backslash\left\{1, \frac{1}{2 \mu+2}, \frac{2 \mu+1}{2 \mu+2}\right\}$ one obtains a Bryant sphere with $2 n$ horospherical smooth ends and Willmore energy $8 n \pi$. The surface on the right in figure 3 is an example corresponding to $n=2, s=t=0.2$.

For $s \in \mathbb{C}^{*}$ and $t=0$ one gets Bryant spheres with one catenoidal and $n$ horospherical ends and Willmore energy $8 n \pi$. The left surface in figure 3 has three smooth ends and corresponds to the parameters $n=2, s=0.2$ and $t=0$, and figure 4 shows two views of the Bryant spheres with 10 smooth ends corresponding to $n=9, s=0.4$ and $t=0$.
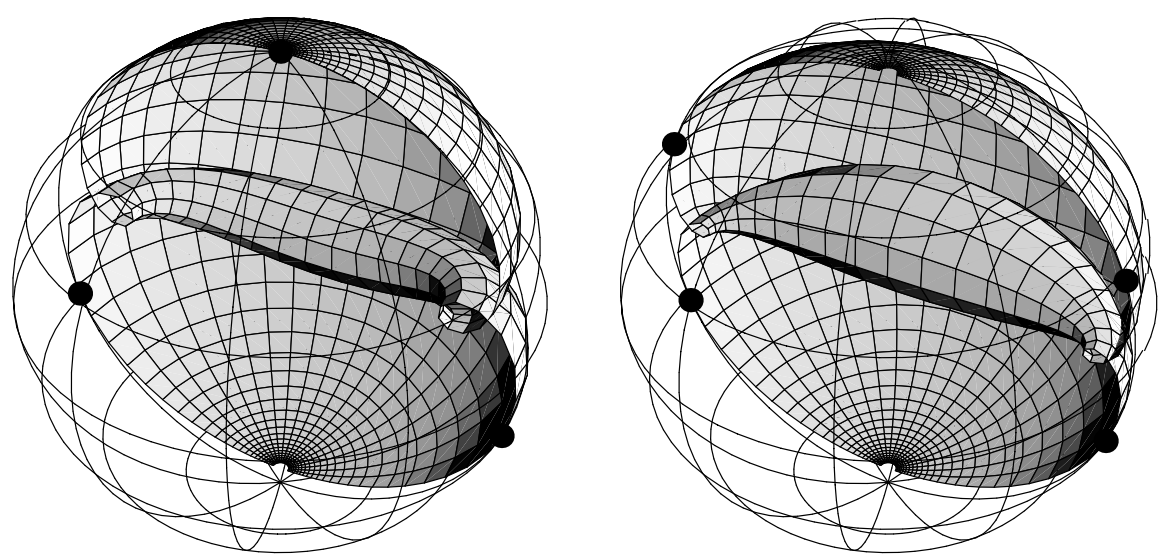

Figure 3: Three- and 4-noid with smooth ends (marked points) and Willmore energy $16 \pi$. 

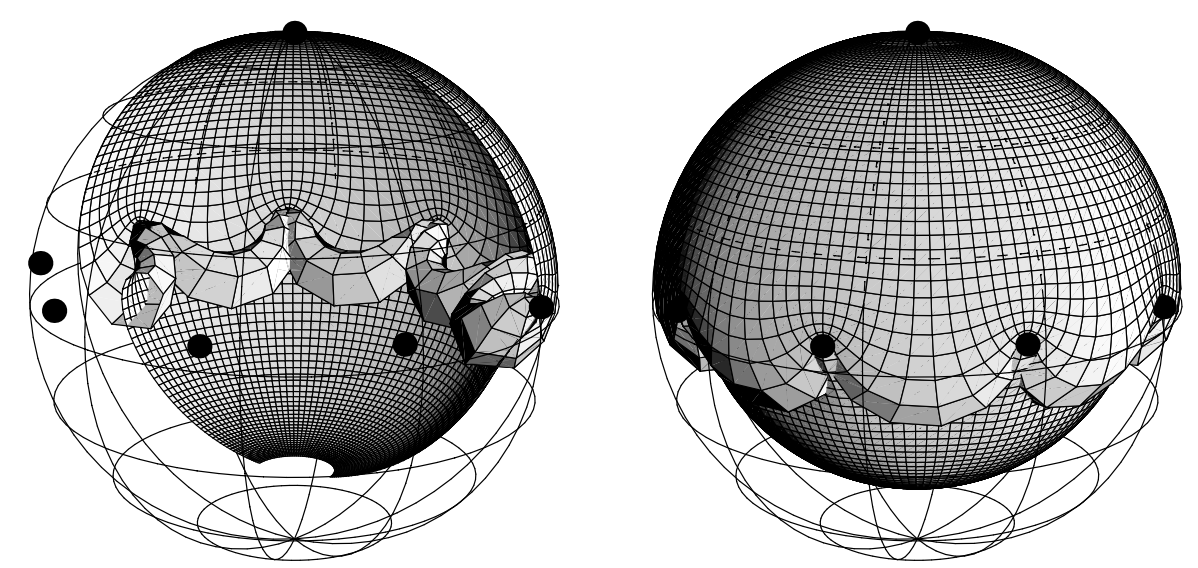

Figure 4: Ten-noid with smooth ends (marked points) and Willmore energy $72 \pi$.

The explicit rational conformal immersions obtained by deforming catenoid cousins with smooth ends show that there exist Bryant spheres with an arbitrary number of smooth ends.

\section{Bryant surfaces as Darboux transforms of the round sphere - a quaternionic approach}

This section is devoted to a Möbius geometric interpretation of the Bryant representation due to Hertrich-Jeromin et al. [15]. For this we use the quaternionic model of Möbius geometry $[10,13,14]$.

The main idea is a Möbius geometric characterization of Bryant surfaces. A conformal immersion into $S^{3}$ is a constant mean curvature \pm 1 surface in the hyperbolic space of curvature -1 with asymptotic boundary $S^{2} \subset S^{3}$ if and only if all its mean curvature spheres are tangent to $S^{2}$, i.e., all its mean curvature spheres are horospheres, cf. figure 5. (This is because horospheres are the spheres with mean curvature \pm 1 in hyperbolic space.) The Bryant representation appears naturally in this context when the hyperbolic Gauss map, which describes the intersection of the mean curvature spheres with the asymptotic boundary, is interpreted as a Darboux transform of the Bryant surface.

The quaternionic approach to four-dimensional Möbius geometry, as introduced in [10], is based on the fact that the quaternionic projective line $\mathbb{H P}^{1}$ with its standard conformal structure inherited from $\mathbb{R}^{4}=\mathbb{H}$ by 

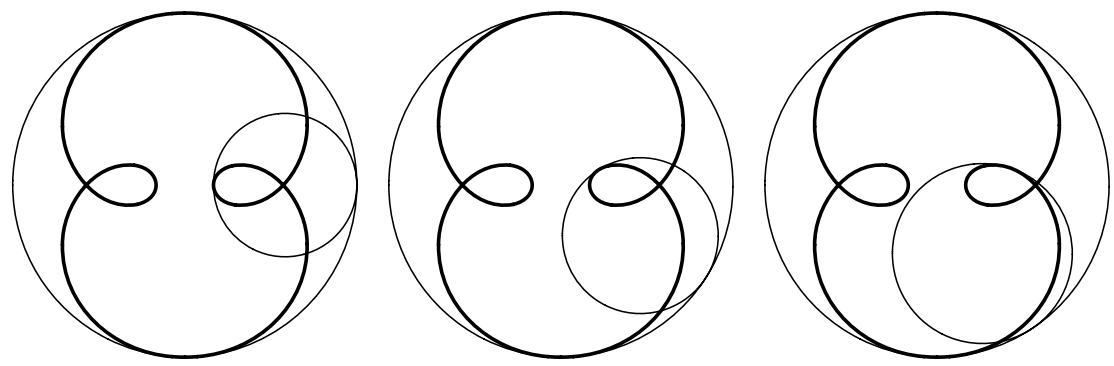

Figure 5: Bryant surface with mean curvature spheres.

the decomposition

$$
\mathbb{H P}^{1}=\{[\lambda, 1] \mid \lambda \in \mathbb{H}\} \cup\{[1,0]\}=\mathbb{H} \cup\{\infty\}
$$

is conformally equivalent to the standard 4-sphere and that, moreover, the group of projective transformations correspond to the group of orientation preserving Möbius transformations. This is a four-dimensional analog to the usual interpretation of $\mathbb{C P}^{1}=\mathbb{C} \cup\{\infty\}$ as the Riemann sphere.

In the following, we identify maps of a Riemann surface $M$ into $\mathbb{H P}^{1}$ with line subbundles $L \subset \mathbb{H}^{2}$ of the trivial quaternionic rank 2 vector bundle $\mathbb{H}^{2}$ over $M$. As explained in [10,4.2], such a map is a conformal immersion if and only if $L$ is an immersed quaternionic holomorphic curve in $\mathbb{H P}^{1}$, which means that the derivative $\delta=\pi d_{\left.\right|_{L}} \in \Omega^{1} \operatorname{Hom}\left(L, \mathbb{H}^{2} / L\right.$ ) of $L$ (with $\pi: \mathbb{H}^{2} \rightarrow \mathbb{H}^{2} / L$ the canonical projection) is nowhere vanishing and satisfies

$$
* \delta=\delta J
$$

for some $J \in \Gamma(\operatorname{End}(L))$ with $J^{2}=-1$ (where $*$ denotes precomposition of the complex structure of $T M)$. Slightly more general, a map $L$ from a Riemann surface into $\mathbb{H}^{1}$ is called a holomorphic curve in $\mathbb{H} \mathbb{P}^{1}$ if it admits $J$ with (4.1).

A fundamental object in Möbius geometric surface theory is the mean curvature sphere congruence of a conformal immersion, which is the unique congruence of oriented touching spheres with the conformally invariant property that pointwise the immersion and the corresponding sphere have the same mean curvature vector. In order to characterize the mean curvature sphere congruence of a holomorphic curve in $\mathbb{H P}^{1}$ in the quaternionic language we need the following description of 2 -spheres $[10,3.4]$ : the oriented 2 -spheres in $\mathbb{H}^{1}$ are in one-to-one correspondence with the quaternionic linear endomorphisms $S \in \operatorname{End}\left(\mathbb{H}^{2}\right)$ with $S^{2}=-$ Id. Such an endomorphism is 
identified with the 2-sphere $\left\{[x] \in \mathbb{H P}^{1} \mid[S x]=[x]\right\}$ which we denote by $S$, too. The endomorphism $S$ induces an orientation of the corresponding 2sphere as it distinguishes a complex structure on the tangent bundle. Hence, $S$ and $-S$ describe the same 2 -sphere with different orientations.

As shown in $[10,5.2]$, the mean curvature sphere congruence of $L$ is the unique section $S \in \Gamma\left(\operatorname{End}\left(\mathbb{H}^{2}\right)\right)$ with $S^{2}=-\operatorname{Id}$ that satisfies

$$
S L=L, \quad * \delta=S \delta=\delta S, \quad \text { and } \quad Q_{\left.\right|_{L}}=0
$$

where $Q=\frac{1}{4}(S d S-* d S)$. The first two conditions describe oriented touching of the immersion and the sphere at the corresponding point while the third condition singles out the mean curvature sphere congruence among all congruences of touching spheres. In the second equation, $S$ stands for the induced complex structures on $\mathbb{H}^{2} / L$ and $L$, in particular, $S$ restricted to $L$ equals the given complex structure $J$ of the holomorphic curve $L$ determined by (4.1). Given the first two conditions in (4.2), the third one is equivalent to $\operatorname{im}(A) \subset L$ where $A=\frac{1}{4}(S d S+* d S)$.

Before we come to the Möbius geometric interpretation of Bryant's representation we need to introduce the notion of Darboux transformation (in the isothermic surface sense): two nowhere intersecting conformal immersions of the same Riemann surface $M$ into the conformal 4-sphere are called a Darboux pair or Darboux transform of each other if there is a family of 2-spheres parametrized by $M$ that touches both immersions at corresponding points with the right orientation. As we will see below (4.5) this definition immediately generalizes to holomorphic curves in $\mathbb{H} \mathbb{P}^{1}$ that are not immersed.

Our treatment of Darboux transformations is in the spirit of $[2,4]$ although we do not deal here with the more general notion of Darboux transformation presented there. Cf. [14] for more information on the Darboux transformation of isothermic surfaces.

Assume $L, L^{\sharp} \subset \mathbb{H}^{2}$ are two conformal immersions of a Riemann surface $M$ into $\mathbb{H}^{1}$ that do not intersect, i.e., $\mathbb{H}^{2}=L \oplus L^{\sharp}$. Because $L^{\sharp} \cong \mathbb{H}^{2} / L$ and $L \cong \mathbb{H}^{2} / L^{\sharp}$ are canonically isomorphic, it makes sense to interpret the derivatives $\delta$ of $L$ and $\delta^{\sharp}$ of $L^{\sharp}$ as 1 -forms with values in $\operatorname{Hom}\left(L, L^{\sharp}\right)$ respectively $\operatorname{Hom}\left(L^{\sharp}, L\right)$. With respect to the splitting $\mathbb{H}^{2}=L \oplus L^{\sharp}$, the trivial connection $d$ of $\mathbb{H}^{2}$ takes the form

$$
d=\left(\begin{array}{cc}
\nabla^{L} & \delta^{\sharp} \\
\delta & \nabla^{\sharp}
\end{array}\right),
$$


where $\nabla^{L}$ and $\nabla^{\sharp}$ are connections induced on $L$ and $L^{\sharp}$. A sphere congruence $\tilde{S}$ that pointwise intersects both $L$ and $L^{\sharp}$ has to be of the form

$$
\tilde{S}=\left(\begin{array}{cc}
J & 0 \\
0 & J^{\sharp}
\end{array}\right)
$$

with $J \in \Gamma(\operatorname{End}(L))$ and $J^{\sharp} \in \Gamma\left(\operatorname{End}\left(L^{\sharp}\right)\right)$. The condition that such a sphere congruence $\tilde{S}$ touches both $L$ and $L^{\sharp}$ with the right orientation is (cf. second condition in $(4.2))$

$$
* \delta=J^{\sharp} \delta=\delta J \quad \text { and } \quad * \delta^{\sharp}=J \delta^{\sharp}=\delta^{\sharp} J^{\sharp} .
$$

In particular, $J$ and $J^{\sharp}$ are the complex structures of the holomorphic curves $L$ and $L^{\sharp}$. Obviously, (4.5) makes sense for non-immersed holomorphic curves as well. In the following, we take (4.5) as the definition of Darboux transformations in the context of (not necessarily immersed) holomorphic curves $L, L^{\sharp}$ with $L \oplus L^{\sharp}=\mathbb{H}^{2}$.

An important characterization of the Darboux transformation is in terms of the retraction form [14]: a retraction form of a holomorphic curve $L$ is a closed 1-form $\omega \in \Omega^{1} \operatorname{End}\left(\mathbb{H}^{2}\right)$ satisfying im $\omega \subset L \subset$ ker $\omega$. For a retraction form $\omega$, the connection $d-\omega$ is flat, because $d \omega=0$ and $\omega \wedge \omega=0$ imply the Maurer-Cartan equation $d \omega=\omega \wedge \omega$. Moreover, at the points where the mean curvature sphere $S$ of $L$ exists, e.g., where $L$ is immersed, $\omega$ satisfies

$$
* \omega=S \omega=\omega S \text {. }
$$

Given two quaternionic holomorphic curves $L, L^{\sharp} \subset \mathbb{H}^{2}$ that form a Darboux pair, a retraction form $\omega \in \Omega^{1} \operatorname{End}\left(\mathbb{H}^{2}\right)$ for $L$ can be, with respect to the splitting $\mathbb{H}^{2}=L \oplus L^{\sharp}$, defined by

$$
\omega=\left(\begin{array}{cc}
0 & \delta^{\sharp} \\
0 & 0
\end{array}\right) .
$$

This retraction form $\omega$ has the property that $L^{\sharp}$ is a parallel subbundle with respect to the flat connection $d-\omega$. Conversely, given a retraction form $\omega$ for $L$, every $(d-\omega)$-parallel line bundle in $\mathbb{H}^{2}$ that does not intersect $L$ is a Darboux transform of $L$ (by (4.3) and (4.6)).

We obtain that, for simply connected $M$, every Darboux transform $L^{\sharp}$ of a holomorphic curve $L$ is of the form

$$
L^{\sharp}=F\left(\begin{array}{c}
\mathbf{k} \\
1
\end{array}\right) \mathbb{H},
$$


where $F: M \rightarrow \mathrm{GL}(2, \mathbb{H})$ is a solution to

$$
d F=\omega F
$$

for some retraction form $\omega \in \Omega^{1} \operatorname{End}\left(\mathbb{H}^{2}\right)$ of $L$. The Darboux transform $L^{\sharp}$ is immersed if and only if $\omega$ has no zeros. The choice of the vector $\left(\begin{array}{l}\mathbf{k} \\ 1\end{array}\right)$ is arbitrary for the present statement, but has the effect that formula (4.12) coincides with formula (2.2).

For the rest of this section we will work with the three-dimensional hyperbolic space defined by fixing the following 2- and 3-sphere in $\mathbb{H P}^{1}$ : $\mathbb{C} \cup\{\infty\} \subset \mathbb{H} \mathbb{P}^{1}$ with corresponding endomorphism $S_{0}=\mathbf{i}$ Id and $\operatorname{Span}_{\mathbb{R}}\{1$, $\mathbf{i}, \mathbf{k}\} \cup\{\infty\} \subset \mathbb{H P}^{1}$, respectively. The hyperbolic space we are working with is thus the Poincaré half space

$$
\mathbf{H}^{3}=\left\{v_{1}+v_{2} \mathbf{i}+v_{3} \mathbf{k} \mid v_{1}, v_{2}, v_{3} \in \mathbb{R}, v_{3}>0\right\} \subset \mathbb{H} \cup\{\infty\}=\mathbb{H}^{1}
$$

We call a surface in $\mathbb{H}^{1}$ a Bryant surface if it is, up to some projective transformation, a Bryant surface in $\mathbf{H}^{3}$ in the usual sense. Note that this includes surface with mean curvature minus one in $\mathbf{H}^{3}$.

The Möbius geometric description of the Bryant representation in terms of Darboux transformations is given by the following theorem.

Theorem 4.1 [15]. A non-totally umbilic surface immersed into $\mathbb{H}^{1}$ is a Bryant surface if and only if it has a totally umbilic Darboux transform. The totally umbilic Darboux transform is then the hyperbolic Gauss map of the Bryant surface.

Note that at a smooth Bryant end the immersion coincides with the hyperbolic Gauss map which therefore ceases to be a Darboux transform, because the splitting $L \oplus L \#$ "collapses".

Proof. Suppose that the conformal immersion $L^{\sharp} \subset \mathbb{H}^{2}$ has a totally umbilic Darboux transform $L \subset \mathbb{H}^{2}$. Because the notion of Darboux transformation is symmetric, $L^{\sharp}$ is also a Darboux transform of $L$. As explained above there is a retraction form $\omega$ of $L$ and a solution $F: \tilde{M} \rightarrow \mathrm{GL}(2, \mathbb{H})$ to $(4.9)$ defined on the universal covering $\tilde{M}$ of $M$ such that $L^{\sharp}=F\left(\begin{array}{c}\mathbf{k} \\ 1\end{array}\right) \mathbb{H}$. We may assume that $L$ is contained in the 2-sphere $S_{0}$ and that for some point $p_{0} \in M$ we have $F_{p_{0}}=\mathrm{Id}$. The mean curvature sphere of $L$ is then $S_{0}$ and, by (4.6), we have $* \omega=S_{0} \omega=\omega S_{0}$. This shows that $\omega$ is a complex holomorphic 1 -form with values in $\operatorname{gl}(2, \mathbb{C})$. Moreover, $\operatorname{im}(\omega) \subset L \subset \operatorname{ker}(\omega)$ implies that $\omega$ is traceless and has vanishing determinant, so $F$ is a holomorphic null immersion into $\mathrm{SL}(2, \mathbb{C})$. 
The immersion $L^{\sharp}$ is either contained in $S_{0}$ or does not intersect $S_{0}$ at all, because for all $\lambda \in \mathbb{H}$ the section $\varphi:=S_{0} F\left(\begin{array}{c}\mathbf{k} \\ 1\end{array}\right)-F\left(\begin{array}{c}\mathbf{k} \\ 1\end{array}\right) \lambda$ is $(d-\omega)$-parallel and therefore vanishes identically or has no zeros at all. Since $L^{\sharp}$ is not totally umbilic, $L^{\sharp}$ and $S_{0}$ do not intersect.

In order to prove that $L^{\sharp}$ is a Bryant surface, we have to show that the immersion $L^{\sharp}$ takes values in $\mathbf{H}^{3}$ and that the sphere congruence $\tilde{S}$ that touches $L$ and $L^{\sharp}$ (see $(4.4)$ ) is the mean curvature sphere of $L^{\sharp}$. To do so we write the holomorphic null immersion $F$ into $\operatorname{SL}(2, \mathbb{C})$ as

$$
F=\left(\begin{array}{ll}
a & b \\
c & d
\end{array}\right): \tilde{M} \rightarrow \mathrm{SL}(2, \mathbb{C}) .
$$

The immersion $L^{\sharp}=F\left(\begin{array}{l}\mathbf{k} \\ 1\end{array}\right) \mathbb{H}$ then becomes

$$
L^{\sharp}=\frac{a \bar{c}+b \bar{d}+\mathbf{k}}{|c|^{2}+|d|^{2}}: M \rightarrow \mathbf{H}^{3},
$$

which is exactly formula (2.2) for the Bryant representation in the half space model. Because $L^{\sharp}$ is defined on $M, F$ has monodromy in $\mathrm{SU}(2)$. This follows from the fact that a $\mathrm{SL}(2, \mathbb{C})$-matrix is in $\mathrm{SU}(2)$ if and only if it leaves the quaternionic line $\left(\begin{array}{l}\mathbf{k} \\ 1\end{array}\right) \mathbb{H}$ invariant.

To show that the 2 -sphere congruence $\tilde{S}$, as given in (4.4), is the mean curvature sphere of $L^{\sharp}$ we have to check that

$$
Q=\frac{1}{4}(\tilde{S} d \tilde{S}-* d \tilde{S})=\left(\begin{array}{cc}
Q^{L} & 0 \\
0 & Q^{\sharp}
\end{array}\right)
$$

vanishes on $L^{\sharp}$, or, equivalently, that $Q^{\sharp}=\frac{1}{4}\left(J^{\sharp} \nabla^{\sharp} J^{\sharp}-* \nabla^{\sharp} J^{\sharp}\right)=0$ where $\nabla^{\sharp}$ denotes the connection on $\operatorname{End}\left(L^{\sharp}\right)$ induced by decomposition (4.3) of the trivial connection $d$. This fact is a consequence of $d S_{0}=0$ : because $S_{0}$ is the mean curvature sphere of $L$, there is a section $H \in \Gamma\left(\operatorname{Hom}\left(L^{\sharp}, L\right)\right)$ such that $S_{0}$, with respect to the splitting $\mathbb{H}^{2}=L \oplus L^{\sharp}$, takes the form

$$
S_{0}=\left(\begin{array}{cc}
J & H \\
0 & J^{\sharp}
\end{array}\right) \quad \text { and therefore } \quad 0=d S_{0}=\left(\begin{array}{cc}
* & * \\
* & \nabla^{\sharp} J^{\sharp}+\delta H
\end{array}\right) .
$$

Hence, $\nabla^{\sharp} J^{\sharp}=-\delta H$ and, by $* \delta=J^{\sharp} \delta$ (see (4.5)), we obtain $Q^{\sharp}=0$.

To prove the converse suppose now that $L^{\sharp}$ is a Bryant surface in $\mathbf{H}^{3}$ and denote by $S$ the mean curvature sphere congruence of $L^{\sharp}$. Then $S_{p}$ is a horosphere for all $p \in M$, i.e., it touches the ideal boundary $S_{0}$. For every $p \in$ $M$, define $L_{p}$ to be the point of intersection of $S_{0}$ and $S_{p}$. This defines a line 
bundle $L$ with $\mathbb{H}^{2}=L \oplus L^{\sharp}$ and it suffices to check that $* \delta \psi=S \delta \psi=\delta S \psi$ for all $\psi \in \Gamma(L)$ : then $L$ is a holomorphic curve with the complex structure induced by $S$, see (4.1), $L$ is totally umbilic, because its image is contained in $S_{0}$, and, by definition (4.5), $L$ is a Darboux transform of $L^{\sharp}$.

Because both 2-spheres $S_{p}$ and $S_{0}$ have the same tangent space at $L_{p}$ we have $S_{\left.p\right|_{L_{p}}}=S_{\left.\right|_{L_{p}}}$ and $S_{p} \equiv S_{0} \bmod L_{p}$ (we may assume that $S_{0}$ and $S_{p}$ touch with the same orientation). Hence $R:=S-S_{0}$ satisfies $\operatorname{ker} R=L=$ $\operatorname{im} R, L$ is smooth, and for $\psi \in \Gamma(L)$ we obtain

$$
d S(\psi)=d(S \psi)-S d \psi=d\left(S_{0} \psi\right)-S d \psi=\left(S_{0}-S\right) d \psi=R \delta \psi
$$

In particular $d S \psi \in \Omega^{1}(L)$. Because $d S=2(* Q-* A)$ and im $A \subset L^{\sharp}$ (with $Q$ and $A$ as in (4.2) and below) $d S \psi \in \Omega^{1}(L)$ implies $d S \psi=2 * Q \psi$. In particular, $* d S \psi=-S d S \psi=d S S(\psi)$ and, using (4.14), $* R \delta \psi=-S R \delta \psi=$ $R \delta S(\psi)$. Because $R$ is nowhere vanishing and anti-commutes with $S$, this proves $* \delta \psi=S \delta \psi=\delta S \psi$.

\section{Soliton spheres and quantization of the willmore energy}

In this section we prove the quantization of the Willmore energy for compact Bryant surfaces with smooth ends and the fact that Bryant spheres with smooth ends are soliton spheres.

As we have seen in Corollary 3.1, a compact Bryant surface with smooth ends is represented by a meromorphic $\mathrm{SL}(2, \mathbb{C})$-valued map $F$ with $\mathrm{SU}(2)$ monodromy on a compact Riemann surface $M$. Although $F$ itself is not defined on $M$, but on its universal covering $\tilde{M}$, the total pole order of $F$ on $M$ is well defined. The following theorem shows that the Willmore energy of a compact Bryant surface is quantized and directly related to this total pole order.

Theorem 5.1. The Willmore energy of a compact Bryant surface with smooth ends is $4 \pi N$ with $N$ the total pole order of $F$ on a fundamental domain.

Proof. We prove this theorem using the quaternionic Plücker formula [13] and thereby introduce the notation needed in the proof of Theorem 5.2. Let $L \subset \mathbb{H}^{2}$ be the holomorphic curve on a compact Riemann surface $M$ that, away from finitely many points, parametrizes the Bryant surface and 
denote by $L^{\sharp} \subset \mathbb{H}^{2}$ its hyperbolic Gauss map ${ }^{1}$, which, by Theorem 4.1 , is a Darboux transform of $L$. By Corollary 3.1, the Bryant representation $F$ of $L$ is a meromorphic $\mathrm{SL}(2, \mathbb{C})$-valued map on $\tilde{M}$ with poles at the ends. In particular, the hyperbolic Gauss map $L^{\sharp}$ extends holomorphically through the ends, because it is the kernel of the meromorphic 1-form $\omega=d F F^{-1}$. The fact that $F$ has $\mathrm{SU}(2)$-monodromy implies that away from the ends

$$
\psi:=F\left(\begin{array}{c}
\mathbf{k} \\
1
\end{array}\right)
$$

is a section of $L$ with quaternionic monodromy.

We prove now that the section $\gamma$ of $L^{-1}=\left(\mathbb{H}^{2}\right)^{*} / L^{\perp}$ with monodromy defined by $\gamma(\psi)=1$ is holomorphic with respect to the unique quaternionic holomorphic structure $D$ on $L^{-1}$, see [13], for which the constant sections of $\left(\mathbb{H}^{2}\right)^{*}$ project to holomorphic sections of $L^{-1}$. This holomorphic structure satisfies $D \pi=(\pi d)^{\prime \prime}$, where $\pi:\left(\mathbb{H}^{2}\right)^{*} \rightarrow L^{-1}=\left(\mathbb{H}^{2}\right)^{*} / L^{\perp}$ denotes the canonical projection and "denotes $\bar{K}$-part of the 1 -form. Let $\hat{\gamma}$ be the unique section of $\left(L^{\sharp}\right)^{\perp} \subset\left(\mathbb{H}^{2}\right)^{*}$ that away from the ends is defined by $\hat{\gamma}(\psi)=1$. Then $\pi \hat{\gamma}=\gamma$ and $d \hat{\gamma}(\psi)=-\hat{\gamma}(d \psi)=-\hat{\gamma}(\omega \psi)=0$, since $\omega$, as a retraction form for $L^{\sharp}$, takes values in $L^{\sharp}$. Hence $d \hat{\gamma}$ takes values in $L^{\perp}$ and $D \gamma=D \pi \hat{\gamma}=(\pi d \hat{\gamma})^{\prime \prime}=0$. The section $\gamma$ extends smoothly through the ends and its vanishing order equals the pole order of $F$ (which can be seen using the normalization of Lemma 3.1, because $\left.\gamma=\left(\pi e_{2}^{*}\right) \bar{z}^{n}(\bar{d}-c \mathbf{k})^{-1}\right)$.

Since $\gamma$ is a globally defined holomorphic section of $L^{-1}$ with monodromy, we can apply the quaternionic Plücker formula [13] to the onedimensional linear system with monodromy spanned by $\gamma$ : let $\nabla$ be the flat connection on $L$ defined away from the ends by $\nabla \psi=0$. Its $\bar{K}$-part $\nabla^{\prime \prime}$ is a quaternionic holomorphic structure on $L$ defined away from the ends, the $\bar{K}$-part of the dual connection on $L^{-1}$ coincides with the usual holomorphic structure $D$, because $\gamma$ is both $D$-holomorphic and $\nabla$-parallel. Therefore, the quaternionic Plücker formula implies that the difference of the Willmore energies of the quaternionic holomorphic line bundles $\left(L^{-1}, D\right)$ and $\left(L, \nabla^{\prime \prime}\right)$ satisfies

$$
W\left(L^{-1}, D\right)-W\left(L, \nabla^{\prime \prime}\right)=4 \pi\left(-\operatorname{deg}\left(L^{-1}\right)+\operatorname{ord}(\gamma)\right)
$$

Here, $\operatorname{ord}(\gamma)=N$ is the vanishing order of $\gamma$ on a fundamental domain. Moreover, we have $\operatorname{deg}\left(L^{-1}\right)=1-g$ with $g$ the genus of $M$, because $L$ is contained in some 3 -sphere in $\mathbb{H}^{1}$ and therefore $L$ is a quaternionic spin

\footnotetext{
${ }^{1}$ In contrast to the proof of Theorem 4.1 the roles of $L$ and $L^{\sharp}$ are interchanged here.
} 
bundle [17]. Finally, $W\left(L, \nabla^{\prime \prime}\right)=0$, because the Hopf field $Q$ of $\nabla^{\prime \prime}$ on $L$ vanishes. (This fact has already been proven in the preceding section: $\nabla$ on $L$ as defined above coincides with the connection induced from $d$ by the splitting $\mathbb{H}^{2}=L \oplus L^{\sharp}$, see (4.3), and because $L^{\sharp}$ is a totally umbilic Darboux transformation, the Hopf field of $\nabla^{\prime \prime}$ vanishes as proven in the paragraph following 4.13.) We obtain

$$
W\left(L^{-1}\right)=4 \pi(g-1+\operatorname{ord}(\gamma))=4 \pi(g-1+N) .
$$

The Willmore energy of $L^{-1}$ satisfies $W\left(L^{-1}\right)=\int_{M}\left(H^{2}-K\right) d A$ where $H$ is the mean curvature, $K$ the Gaussian curvature, and $d A$ the area element with respect to the Euclidean geometry of $\mathbb{R}^{3} \cong \operatorname{Span}_{\mathbb{R}}\{1, \mathbf{i}, \mathbf{k}\} \subset \mathbb{H}$, see [13]. Assuming that none of the ends lies at $\infty$ of $\mathbb{R}^{3}$, the Gauss-Bonnet Theorem and (5.1) imply

$$
W=\int_{M} H^{2} d A=\int_{M}\left(H^{2}-K\right) d A+4 \pi(1-g)=4 \pi \operatorname{ord}(\gamma)=4 \pi N .
$$

For a Bryant sphere with smooth ends $F$ is rational, so (2.1) shows that Bryant spheres with smooth ends admit conformal parametrizations in terms of rational functions. This is a fundamental property of soliton spheres $[5,18]$. A Soliton sphere is a conformal immersion $L \subset \mathbb{H}^{2}$ of $\mathbb{C P}^{1}$ into $\mathbb{H} \mathbb{P}^{1}$ such that the linear system $\left(\mathbb{H}^{2}\right)^{*} \subset H^{0}\left(L^{-1}\right)$ whose elements are the homogeneous coordinates of $L$, the so-called canonical linear system, is contained in a linear system with equality in the quaternionic Plücker estimate: for an $(n+$ 1)-dimensional linear system $H \subset H^{0}\left(L^{-1}\right)$ of a quaternionic holomorphic line bundle $L^{-1}$ over a compact Riemann surface of genus $g$ the Plücker estimate [13] states that the Willmore energy of $L^{-1}$ satisfies

$$
\frac{1}{4 \pi} W\left(L^{-1}\right) \geq(n+1)\left(n(1-g)-\operatorname{deg} L^{-1}\right)+\operatorname{ord}(H) .
$$

If $L$ lies in some 3 -sphere in $\mathbb{H}^{1}$, then $\operatorname{deg} L^{-1}=1-g$, cf. [17], and

$$
\frac{1}{4 \pi} W\left(L^{-1}\right) \geq\left(n^{2}-1\right)(1-g)+\operatorname{ord}(H) .
$$

The above definition of soliton spheres is a generalization of the one Iskander Taimanov, motivated by the soliton theory of the $\mathrm{mKdV}$ equation, gives for spheres with special symmetry [24]. In [5] we prove that all Willmore spheres, 
i.e., all spheres obtained from complete minimal surfaces of finite total curvature with planar ends, are soliton spheres. We conclude the present article with a proof of the analogous result that all Bryant spheres with smooth ends are soliton spheres. We actually prove a more general statement for compact Bryant surfaces with smooth ends of arbitrary genus.

Theorem 5.2. The canonical linear system of a non-totally umbilic compact Bryant surface with smooth ends is contained in a three-dimensional linear system with monodromy that has equality in the quaternionic Plücker estimate. In particular, Bryant spheres with smooth ends are soliton spheres.

Proof. We proceed using the notation of the proof of Theorem 5.1. Denote by $\left(\mathbb{H}^{2}\right)^{*} \subset H^{0}\left(L^{-1}\right)$ the canonical linear system. Define $\tilde{H}$ to be the threedimensional linear system with monodromy obtained by taking the span of $\left(\mathbb{H}^{2}\right)^{*}$ and the holomorphic section $\gamma$ defined in the proof of Theorem 5.1. The theorem is proven if we show that $\tilde{H}$ has equality in the quaternionic Plücker formula.

Denote by $G: M \rightarrow S_{0}=\mathbb{C} \cup\{\infty\} \subset \mathbb{H} \cup\{\infty\}$, or, projectively $L^{\sharp}=$ $\left[\begin{array}{c}G \\ 1\end{array}\right]$, the hyperbolic Gauss map of $L$ and by $b(G)$ its total branching order. The zeros of $\gamma$ are the ends of the Bryant surface and Lemmas 5.1 and 5.2 below show that the Weierstrass order $\operatorname{ord}(\tilde{H})$ of $\tilde{H}$ is given by

$$
\operatorname{ord}(\tilde{H})=\operatorname{ord}(\gamma)-2 \# \mathrm{ends}+b(G)
$$

The derivative $\delta^{\sharp} \in \Gamma\left(\operatorname{Hom}\left(L^{\sharp}, K \mathbb{H}^{2} / L^{\sharp}\right)\right)$ is linear with respect to the complex structures induced by $S_{0}$ (see (4.5)) and it is complex holomorphic (see [10]). Similarly, the 1 -form $\omega=d F F^{-1}$ is a complex meromorphic section of $\Gamma\left(\operatorname{Hom}\left(\mathbb{H}^{2} / L^{\sharp}, K L^{\sharp}\right)\right.$ ) (see the proof of Theorem 4.1). Hence $\delta^{\sharp} \omega$ is a complex meromorphic quadratic differential on $M$, i.e., a meromorphic section of $K^{2}$. By definition, $\operatorname{ord}\left(\delta^{\sharp}\right)=b(G)$ and, because $\omega$ has second-order poles at the ends (cf. Lemma 3.2) and no zeros, ord $(\omega)=-2$ \#ends. Using $\operatorname{deg}\left(K^{2}\right)=4(g-1)$, this implies

$$
4(g-1)=\operatorname{ord}\left(\delta^{\sharp} \omega\right)=b(G)-2 \# \text { ends }
$$

and, together with (5.1) and 5.3, we obtain

$$
\frac{1}{4 \pi} W\left(L^{-1}\right)=3(1-g)+\operatorname{ord}(\tilde{H})=\left(n^{2}-1\right)(1-g)+\operatorname{ord}(\tilde{H}),
$$

where $n+1=\operatorname{dim} \tilde{H}=3$. Thus for $\tilde{H}$ equality holds in (5.2). 
The following two lemmas are needed to compute the total order (5.3) of the linear system $\tilde{H}$.

Lemma 5.1. If $p \in M$ is not an end, then $\operatorname{ord}_{p}(\tilde{H})=b_{p}(G)$.

Proof. In the proof of Theorem 5.1 we have, away from the ends, defined the section $\hat{\gamma}$ of $\left(L^{\sharp}\right)^{\perp}$. It satisfies $\pi \hat{\gamma}=\gamma$ and $\pi d \hat{\gamma}=0$ for the canonical projection $\pi:\left(\mathbb{H}^{2}\right)^{*} \rightarrow\left(\mathbb{H}^{2}\right)^{*} / L^{\perp}$. This means that $\hat{\gamma}$ can be interpreted as the unique prolongation of the holomorphic section $\gamma$ to the 1-jet bundle of $L^{-1}$, cf. [13].

We chose $\hat{\alpha} \in L_{p}^{\perp}$ and $\hat{\beta}=\hat{\gamma}_{p} \in\left(L^{\sharp}\right)_{p}^{\perp}$. Then $\alpha=\pi \hat{\alpha}$ and $\beta=\pi \hat{\beta}$ form a basis of the canonical linear system $\left(\mathbb{H}^{2}\right)^{*}$. Because $\beta_{p}=\gamma_{p}$, the section $\beta$ does not vanish at $p$ and there are quaternion valued functions $f$ and $h$ defined in a neighborhood of $p$ such that $\alpha=\beta f$ and $\gamma=\beta h$. The sections $\alpha$ and $\tilde{\gamma}=\gamma-\beta=\beta(h-1)$ both vanish at $p$. The section $\alpha$ vanishes to first order at $p$, since $\alpha$ and $\beta$ form a basis of the linear system $\left(\mathbb{H}^{2}\right)^{*}$, which has no Weierstrass points, because $L$ is immersed. In particular, $d_{p} f \neq 0$.

In order to relate the vanishing order of $\tilde{\gamma}$ at $p$ to the branching order of the hyperbolic Gauss map $G$ at $p$, we use that $\hat{\gamma}=\hat{\beta} h+(\hat{\alpha}-\hat{\beta} f) \lambda$ for a quaternion valued function $\lambda$, since $\hat{\alpha}-\hat{\beta} f \in \Gamma\left(L^{\perp}\right)$. By definition of $\hat{\beta}$, we have $\lambda_{p}=0$. Since $d \hat{\gamma}$ takes values in $L^{\perp}$, the equation

$$
d \hat{\gamma}=\hat{\beta} d h-\hat{\beta} d f \lambda+(\hat{\alpha}-\hat{\beta} f) d \lambda
$$

implies $d h=d f \lambda$ and $d \hat{\gamma}=(\hat{\alpha}-\hat{\beta} f) d \lambda$. For the vanishing order of $\tilde{\gamma}=\beta(h-$ 1) at $p$ we therefore obtain (using $d h=d f \lambda, d_{p} f \neq 0$ and $\lambda_{p}=0$ )

$$
\operatorname{ord}_{p}(\tilde{\gamma})=\operatorname{ord}_{p}(d h)+1=\operatorname{ord}_{p}(\lambda)+1=\operatorname{ord}_{p}(d \lambda)+2 \text {. }
$$

For the branching order of $L^{\sharp}$ we obtain, using $d \hat{\gamma}=(\hat{\alpha}-\hat{\beta} f) d \lambda$ and $\hat{\gamma}_{p} \neq 0$,

$$
b_{p}(G)=\operatorname{ord}_{p}(d \hat{\gamma})=\operatorname{ord}_{p}(d \lambda)
$$

The Weierstrass gap sequence of the linear system $\tilde{H}$ for the point $p$ is 0,1 , $b_{p}(G)+2$ (realized by the sections $\left.\beta, \alpha, \tilde{\gamma}\right)$.

Lemma 5.2. If $p \in M$ is an end, then $\operatorname{ord}_{p}(\tilde{H})=\operatorname{ord}_{p}(\gamma)-2+b_{p}(G)$.

Proof. Let $z: U \rightarrow \Delta$ be a holomorphic coordinate on an open neighborhood $U \subset M$ of $p$ such that $z(p)=0$. Let $F=z^{-n}\left(\begin{array}{ll}a & b \\ c & d\end{array}\right)$ be the holomorphic null immersion representing the Bryant surface $L_{\left.\right|_{\Delta^{*}}}$ and suppose that $F$ has the 
normal form of Lemma 3.1. Then $\operatorname{ord}_{0}(\gamma)=n \neq 0$. The hyperbolic Gauss map $L^{\sharp} \subset \mathbb{H}^{2}$ is given by the image of $\omega=F^{\prime} F^{-1} d z$, see Section 4 . Thus

$$
L^{\sharp}=\left(\begin{array}{c}
z^{-n-1}\left(z b^{\prime}-n b\right) \\
z^{-n-1}\left(z d^{\prime}-n d\right)
\end{array}\right) \mathbb{H}=\left(\begin{array}{c}
z b^{\prime}-n b \\
z d^{\prime}-n d
\end{array}\right) \mathbb{H} .
$$

Since $\operatorname{ord}_{0}(b)=1$ (see the proof of Lemma 3.2) and $d(0) \neq 0$, it follows that either $n \geq 2$ and $b_{0}(G)=0$ or $n=1$ and $b_{0}(G)=\operatorname{ord}_{0}\left(b-b^{\prime}(0) z\right)-1 \geq 1$. In the first case the claimed formula follows, since $L$ is immersed and therefore $\left(\mathbb{H}^{2}\right)^{*}$ is Weierstrass point free.

To see the formula in the case $n=1$, let $\alpha, \beta \in\left(\mathbb{H}^{2}\right)^{*} \subset H^{0}\left(L^{-1}\right)$ be the projections to the first and second coordinate of $\mathbb{H}^{2}$. The section $\beta$ does not vanish at $z=0$, because $F$ is in normal form and therefore $L=[\psi]=$ $\left[\begin{array}{l}z^{-1} a \mathbf{k}+z^{-1} b \\ z^{-1} c \mathbf{k}+z^{-1} d\end{array}\right]$ is $\left[\begin{array}{l}0 \\ 1\end{array}\right]$ for $z=0$. The section $\gamma$ vanishes to order 1 . We now compute the vanishing order of $\alpha-\gamma \overline{b^{\prime}(0)}$. We have

$$
\alpha=\beta \bar{z}(\overline{c \mathbf{k}+d})^{-1}(\overline{a \mathbf{k}+b}) \bar{z}^{-1} \quad \text { and } \quad \gamma=\beta \bar{z}(\overline{c \mathbf{k}+d})^{-1} \text {, }
$$

where the last formula follows from

$$
\gamma\left(\begin{array}{c}
z^{-1} a \mathbf{k}+z^{-1} b \\
z^{-1} c \mathbf{k}+z^{-1} d
\end{array}\right)=\gamma(\psi)=1
$$

Thus $\alpha-\gamma \overline{b^{\prime}(0)}=\beta \bar{z}(\overline{c \mathbf{k}+d})^{-1}\left(\overline{a \mathbf{k}+b-b^{\prime}(0) z}\right) \bar{z}^{-1}$ and, by $d(0) \neq 0$, we have $\operatorname{ord}_{0}\left(\alpha-\gamma \overline{b^{\prime}(0)}\right)=\min \left(\operatorname{ord}_{0}(a), \operatorname{ord}_{0}\left(b-b^{\prime}(0) z\right)\right)$. By Lemma 3.1 (and its proof) $a^{\prime}(0)=0$ and $a^{\prime} d^{\prime}-b^{\prime} c^{\prime}=1$, which shows $b^{\prime}(0) c^{\prime}(0)=-1$. Using $b^{\prime}(0) c^{\prime}(0)=-1$, the identities $a^{\prime} d^{\prime}-b^{\prime} c^{\prime}=1$ and $a d-b c=z^{2}$ imply $\operatorname{ord}_{0}(a) \geq \operatorname{ord}_{0}\left(b-b^{\prime}(0) z\right)$. This proves the claim, because then $\operatorname{ord}_{0}(\alpha-$ $\left.\gamma \overline{b^{\prime}(0)}\right)=\operatorname{ord}_{0}\left(b-b^{\prime}(0) z\right)=b_{0}(G)+1$. The Weierstrass order at $p$ is therefore $\operatorname{ord}(\tilde{H})_{p}=b_{0}(G)-1$, since the Weierstrass gap sequence at zero is $0,1, b_{0}(G)+1$ (realized by $\left.\beta, \gamma, \alpha-\gamma \overline{b^{\prime}(0)}\right)$.

Remark 5.1. At the beginning of the proof of Lemma 5.2 we have seen that the hyperbolic Gauss map is immersed at a smooth end if and only if the pole order of $F$ at the end is greater than 1, i.e., if the end is catenoidal and not horospherical in the sense of [25].

\section{Bryant's quartic differential}

We show that the vanishing of Bryant's quartic differential $\mathcal{Q}$ provides a uniform Möbius geometric characterization of the compact immersed surfaces 
$f: M \rightarrow S^{3}$ in the conformal 3-sphere $S^{3}$ that can be obtained as compactification of either a Euclidean minimal surface with planar ends or a Bryant surfaces with smooth ends.

The quartic differential $\mathcal{Q}$ was introduced by Bryant [7] for Willmore surfaces in the conformal 3-sphere $S^{3}$. It was pointed out by Konrad Voss in a talk given at Oberwolfach that $\mathcal{Q}$ may be defined for arbitrary conformal immersions of a Riemann surface $M$ into $S^{3}$ and that $\mathcal{Q}$ is holomorphic if and only if, locally and away from umbilics and isolated points, the immersion is Willmore or has constant mean curvature with respect to some space form geometry, see [3] for a proof. We prove the following global characterization of compact surfaces with $\mathcal{Q} \equiv 0$.

Theorem 6.1. Let $f: M \rightarrow S^{3}$ be a conformal immersion of a compact Riemann surface $M$ into the conformal 3-sphere $S^{3}$. The quartic differential $\mathcal{Q}$ of $f$ vanishes identically if and only if $f$ is the compactification of either a Euclidean minimal surface with planar ends or a Bryant surface with smooth ends.

Here an immersion $f: M \rightarrow S^{3}$ into the conformal 3-sphere is called a Euclidean minimal surface if and only if it admits a point $\infty \in S^{3}$ not on $f$ in which all its mean curvature spheres intersect, i.e., if the resulting immersion into $\mathbb{R}^{3}=S^{3} \backslash\{\infty\}$ is Euclidean minimal. An immersion is the compactification of a Euclidean minimal surface with planar ends if and only if the resulting immersion into $\mathbb{R}^{3}=S^{3} \backslash\{\infty\}$ is a complete Euclidean minimal surface with finite total curvature and planar ends, see [7]. Similarly, an immersion $f: M \rightarrow S^{3}$ into the conformal 3-sphere is a Bryant surface if and only if it has a totally umbilic darboux transform, cf. Theorem 4.1.

Proof. Lemma 6.1 below implies that $f$ has only finitely many umbilics. The theorem then follows from Proposition 6.1.

Using that a holomorphic quartic differential on the sphere vanishes identically and that, by Bryant [7], every Willmore spheres is the compactification of a Euclidean minimal surface with planar ends, we obtain:

Corollary 6.1. Let $f: \mathbb{C P}^{1} \rightarrow S^{3}$ be a conformal immersion of the 2-sphere into the 3-sphere. The quartic differential $\mathcal{Q}$ of $f$ is holomorphic if and only if $f$ is a Willmore sphere or a Bryant sphere with smooth ends.

For the proof of Lemma 6.1 and Proposition 6.1 we use the light cone model of the conformal 3-sphere and the invariants introduced in [11]. The 
light cone model of the conformal 3 -sphere $S^{3}$ is based on the identification

$$
S^{3} \cong \mathbb{P}(\mathcal{L}), \quad x \leftrightarrow[1: x]
$$

of $S^{3} \subset \mathbb{R}^{4}$ with the projectivized light cone $\mathcal{L} \subset \mathbb{R}^{4,1}$ in five-dimensional Minkowski space $\mathbb{R}^{4,1}$ with the metric $\langle x, x\rangle=-x_{0}^{2}+\sum_{i=1}^{4} x_{i}^{2}$. Under this identification, the group of Möbius transformations of $S^{3}$ is identified with the identity component of the group of linear transformation that preserve the Minkowski product $\langle$,$\rangle .$

Let $f: M \rightarrow S^{3}$ be a conformal immersion of a Riemann surface $M$ into the conformal 3-sphere and $z: M \supset U \rightarrow \mathbb{C}$ a holomorphic chart on $M$. Then there is a unique holomorphic lift $\psi: U \rightarrow \mathcal{L}$ of $f$ such that

$$
\langle d \psi, d \psi\rangle=|d z|^{2}
$$

and $\psi_{0}>0$. Denote $\gamma: M \rightarrow \mathbb{R}^{4,1}$ the unique (up to sign) smooth map with

$$
\gamma \perp\left\{\psi, \psi_{z}, \psi_{\bar{z}}, \psi_{z \bar{z}}\right\} \quad \text { and } \quad\langle\gamma, \gamma\rangle=1
$$

Then $\mathbb{P}\left(\gamma^{\perp} \cap \mathcal{L}\right)$ is the mean curvature sphere congruence or conformal Gauss map of $f$. The conformal Hopf differential and Schwarzian derivative of $f$ with respect to $z$ are the smooth functions $\kappa, c: U \rightarrow \mathbb{C}$ satisfying

$$
\psi_{z z}+\frac{c}{2} \psi=\kappa \gamma
$$

The corresponding functions $\tilde{\kappa}, \tilde{c}$ with respect to another holomorphic chart $\tilde{z}: U \rightarrow \mathbb{C}$ are given by

$$
\tilde{\kappa} \frac{d \tilde{z}^{2}}{|d \tilde{z}|}=\kappa \frac{d z^{2}}{|d z|} \quad \text { and } \quad \tilde{c} d \tilde{z}^{2}=\left(c-S_{z}(\tilde{z})\right) d z^{2}
$$

where $S_{z}(\tilde{z})=\left(\frac{\tilde{z}_{z z}}{\tilde{z}_{z}}\right)_{z}-\frac{1}{2}\left(\frac{\tilde{z}_{z z}}{\tilde{z}_{z}}\right)^{2}$ is the usual Schwarzian derivative.

Denote $\hat{\psi}: U \rightarrow \mathcal{L}$ the unique map such that the Minkowski product takes the form

$$
\left(\begin{array}{ccccc}
0 & 0 & 0 & -1 & 0 \\
0 & 0 & \frac{1}{2} & 0 & 0 \\
0 & \frac{1}{2} & 0 & 0 & 0 \\
-1 & 0 & 0 & 0 & 0 \\
0 & 0 & 0 & 0 & 1
\end{array}\right)
$$

with respect to the frame $\left(\psi, \psi_{z}, \psi_{\bar{z}}, \hat{\psi}, \gamma\right)$. The frame equations are then

$$
\begin{aligned}
\psi_{z z} & =-\frac{c}{2} \psi+\kappa \gamma, & \psi_{z \bar{z}} & =-|\kappa|^{2} \psi+\frac{1}{2} \hat{\psi}, \\
\hat{\psi}_{z} & =-2|\kappa|^{2} \psi_{z}-c \psi_{\bar{z}}+2 \kappa_{\bar{z}} \gamma, & \gamma_{z} & =2 \kappa_{\bar{z}} \psi-2 \kappa \psi_{\bar{z}}
\end{aligned}
$$


with compatibility conditions

$$
\begin{array}{ll}
\frac{1}{2} c_{\bar{z}}=2\left(|\kappa|^{2}\right)_{z}+2 \bar{\kappa}_{z} \kappa & \text { (Gauss equation) } \\
\operatorname{Im}\left(\kappa_{\bar{z} \bar{z}}+\frac{\bar{c}}{2} \kappa\right)=0 & \text { (Codazzi equation) }
\end{array}
$$

In particular, the second $z$-derivative of $\gamma$ is

$$
\gamma_{z z}=\left(2 \kappa_{z \bar{z}}+2 \kappa|\kappa|^{2}\right) \psi+2 \kappa_{\bar{z}} \psi_{z}-2 \kappa_{z} \psi_{\bar{z}}-\kappa \hat{\psi}
$$

Bryant's quartic differential is defined as

$$
\mathcal{Q}=\frac{1}{4}\left\langle\gamma_{z z}, \gamma_{z z}\right\rangle d z^{4}=\left(\kappa \kappa_{\bar{z} z}+\kappa^{2}|\kappa|^{2}-\kappa_{\bar{z}} \kappa_{z}\right) d z^{4}
$$

(since $\left(\psi, \psi_{x}, \psi_{y}, \gamma, \hat{\psi}\right)$ with $z=x+i y$ is a local section of $\mathcal{F}_{f}^{(\gamma)}$ in $[7$, Theorem B]).

The umbilic points of $f$ are those points at which the mean curvature sphere touches $f$ to second order, i.e., umbilic points are the zeros of $\kappa$. On the complement $M_{0}$ of the set of umbilic points of $f$, its mean curvature sphere congruence has a unique second envelope $f^{\sharp}: M_{0} \rightarrow S^{3}$ which is defined by the property that any lift $\psi^{\sharp}$ of $f^{\sharp}$ satisfies

$$
\left\langle\psi^{\sharp}, \psi^{\sharp}\right\rangle=\left\langle\psi^{\sharp}, \gamma\right\rangle=\left\langle\psi^{\sharp}, d \gamma\right\rangle=0 .
$$

With respect to a chart $z$, a lift of $f^{\sharp}$ can be obtained by

$$
\psi^{\sharp}=2\left|\kappa_{\bar{z}}\right|^{2} \psi-2 \kappa_{\bar{z}} \bar{\kappa} \psi_{z}-2 \bar{\kappa}_{z} \kappa \psi_{\bar{z}}+|\kappa|^{2} \hat{\psi} .
$$

Lemma 6.1. A conformal immersion $f: M \rightarrow S^{3}$ of a compact Riemann surface $M$ into the 3-sphere whose quartic differential $\mathcal{Q}$ vanishes identically is either totally umbilic or has only finitely many umbilic points.

Proof. The idea of the proof is to define a complete metric $g$ on the complement $M_{0}$ of the set of umbilics that has non-positive curvature and finite total curvature. From [16, Chapter III, Proposition 16] we then obtain that $M \backslash M_{0}$ consists of a finite number of points. In order to define the metric $g$ we use the Uniformization Theorem and distinguish the cases that $M$ has genus 0,1 , or greater than 1 .

1. Case $M=\mathbb{C P}^{1}$ : without loss of generality, we can assume that $\infty \in$ $\mathbb{C P}^{1}$ is an umbilic point, i.e., $\infty \notin M_{0}$. Denote $\kappa$ the conformal Hopf 
differential with respect to a holomorphic chart $z: \mathbb{C P}^{1} \backslash\{\infty\} \rightarrow \mathbb{C}$. Then

$$
g=e^{2 u}|d z|^{2}, \quad u=-\log |\kappa|
$$

defines a (coordinate dependent) metric on $M_{0}$. By (6.2) we have $g=$ $\frac{|d \tilde{z}|^{2}}{|\tilde{\kappa}|^{2}|\tilde{z}|^{8}}$ for $\tilde{z}=\frac{1}{z}$. Thus $g$ is complete, because $\tilde{\kappa}$ is smooth near $\infty, \kappa$ is smooth on $\mathbb{C P}^{1} \backslash\{\infty\}$, and zero on $\mathbb{C P}^{1} \backslash M_{0}$. The curvature of $g$ is

$$
K=-4 e^{-2 u} u_{z \bar{z}}=4|\kappa|^{2} \operatorname{Re}\left(\frac{\kappa_{\bar{z} z} \kappa-\kappa_{z} \kappa_{\bar{z}}}{\kappa^{2}}\right)=-4|\kappa|^{4},
$$

where the last equality holds because $\mathcal{Q} \equiv 0$ in 6.7 . The total curvature of $g$ is finite, because

$$
\int_{M_{0}} K d A=-4 \int_{\mathbb{C}}|\kappa|^{2} d x \wedge d y=-4 \tilde{W}
$$

where $W=\tilde{W}+2 \pi \chi(M)$ is the Willmore energy of $f$, cf. [11, Section 3.2] and Appendix A.

2. Case $M=\mathbb{C} / \Gamma$ : the conformal Hopf differential $\kappa$ with respect to the chart $z$ on the universal cover $\mathbb{C}$ is invariant under the translations by elements in the lattice $\Gamma$ and defines a function $\kappa: M \rightarrow \mathbb{C}$. Hence as in the genus 0 case

$$
g=e^{2 u}|d z|^{2}, \quad u=-\log |\kappa|
$$

is a metric on $M_{0}$ with the desired properties.

3. Case $M=\mathbf{B}^{2} / \Gamma$ for $\mathbf{B}^{2} \subset \mathbb{C}$ the unit disc with the Poincaré metric $\hat{g}=\frac{4|d z|^{2}}{\left(1-|z|^{2}\right)^{2}}$ and $\Gamma$ a discrete group of isometries of $\mathbf{B}^{2}$ : now $\kappa$ with respect to the chart $z$ on the universal cover $\mathbf{B}^{2}$ is not invariant under $\Gamma$, but

$$
|\kappa|\left(1-|z|^{2}\right): M \rightarrow \mathbb{R}
$$

is well defined by (6.2), because $\Gamma$ is a group of isometries of $\hat{g}$. Thus

$$
g=e^{2 u}|d z|^{2}=\frac{\hat{g}}{|\kappa|^{2}\left(1-|z|^{2}\right)^{2}}, \quad u=-\log \left|\frac{1}{2} \kappa\left(1-|z|^{2}\right)^{2}\right|
$$

defines a complete metric on $M_{0}$ with non-positive curvature

$$
K=-4 e^{-2 u} u_{z \bar{z}}=-|\kappa|^{4}\left(1-|z|^{2}\right)^{4}-2|\kappa|^{2}\left(1-|z|^{2}\right)^{2} .
$$


The total curvature of $g$ is finite, because

$$
\int_{M_{0}} K d A=-4 \int_{\Delta}|\kappa|^{2} d x \wedge d y-2 \int_{\Delta} \frac{4}{\left(1-|z|^{2}\right)^{2}} d x \wedge d y=-4 \tilde{W}-2 \hat{A}
$$

where $\Delta \subset \mathbf{B}^{2}$ is a fundamental domain of $\Gamma$ and $\hat{A}$ its hyperbolic area.

The following proposition is a local version of Theorem 6.1 which holds away from umbilic points.

Proposition 6.1. Let $f: M \rightarrow S^{3}$ be an umbilic free conformal immersion of a Riemann surface $M$ into the conformal 3-sphere $S^{3}$. The quartic differential $\mathcal{Q}$ of $f$ vanishes identically if and only if either $f$ is Euclidean minimal in $\mathbb{R}^{3}=S^{3} \backslash\{\infty\}$ for some $\infty \in S^{3}$ or $f$ is a Bryant surface.

Proof. By (6.3) and (6.7), the derivative of $\psi^{\sharp}$ in (6.9) is

$$
\psi_{z}^{\sharp}=\kappa^{-1} \kappa_{z} \psi^{\sharp}+\lambda \bar{\kappa} \gamma_{z}+\kappa^{-1} q \gamma_{\bar{z}},
$$

where

$$
\lambda=\bar{\kappa}^{-1} \bar{\kappa}_{z z}+\frac{c}{2} \quad \text { and } \quad \mathcal{Q}=q d z^{4} .
$$

If $\mathcal{Q} \equiv 0$ then $\lambda$ vanishes at a point if and only if the second envelope $f^{\sharp}$ of the mean curvature sphere congruence of $f$ is not immersed at this point. The latter property is clearly independent of the chart. Because

$$
\lambda_{\bar{z}}=\bar{\kappa}^{-2}\left(\bar{\kappa} \bar{\kappa}_{\bar{z} z}+\bar{\kappa}^{2}|\kappa|^{2}-\bar{\kappa}_{\bar{z}} \bar{\kappa}_{z}\right)_{z}=\bar{\kappa}^{-2}(\bar{q})_{z}=0
$$

the function $\lambda$ is holomorphic and hence either vanishes identically or has isolated zeros. So $f^{\sharp}$ is either constant or, away from isolated points, an immersion. In the first case $f$ is Euclidean minimal in $\mathbb{R}^{3}=S^{3} \backslash\{\infty\}$ for $\infty=f^{\sharp}$ (note that $f$ does not go through $\infty$, see $(6.9)$ ).

In case $f^{\sharp}$ is non-constant we assume for a moment that it is globally immersed. Then $f^{\sharp}$ is conformal, because $\left\langle\psi_{z}^{\sharp}, \psi_{z}^{\sharp}\right\rangle=0$ by 6.8 and (6.10), and $\left\langle\gamma_{z}, \gamma_{z}\right\rangle=0$ which follows from 6.3. Taking the derivative in (6.10) shows that $\psi_{z z}^{\sharp}$ lies in the span of $\psi^{\sharp}, \gamma_{z}$, and $\gamma_{z z}$. Since $\mathcal{Q} \equiv 0$, equations $(6.7)$ and 6.8 imply that the complex subspace spanned by $\psi^{\sharp}, \gamma_{z}$, and $\gamma_{z z}$ is a null space for the non-degenerate symmetric product induced by the Minkowski metric. But a null space for a non-degenerate symmetric product on a fivedimensional space is at most two-dimensional such that $\psi^{\sharp}, \psi_{z}^{\sharp}$, and $\psi_{z z}^{\sharp}$ are linearly dependent and $f^{\sharp}$ is totally umbilic. In other words, the second 
envelope $f^{\sharp}$ is a holomorphic map into a round 2-sphere in $S^{3}$. By continuity, this more generally holds in case $f^{\sharp}$ is globally immersed except for isolated points.

Using (6.10) one can check that away from the branch points of $f^{\sharp}$ the frame $\left(\psi, \psi_{x}, \psi_{y}, \gamma, \hat{\psi}\right)$ induces the same orientation as $\left(\psi^{\sharp}, \psi_{x}^{\sharp}, \psi_{y}^{\sharp}, \gamma, \hat{\psi}\right)$, i.e., the envelopes $f^{\sharp}$ and $f$ of the mean curvature sphere congruence of $f$ touch with the same orientation. Hence $f^{\sharp}$ is a totally umbilic Darboux transform of $f$ and $f$ is a Bryant surface by Theorem 4.1 .

Conversely, if $f$ is Euclidean minimal then $f^{\sharp}$ is constant and (6.10) implies $\mathcal{Q} \equiv 0$. If $f$ is a Bryant surface then Theorem 4.1 implies that $f^{\sharp}$ is a totally umbilic branched conformal immersion, such that

$$
0=\left\langle\psi_{z}^{\sharp}, \psi_{z}^{\sharp}\right\rangle=4 \bar{\kappa}^{2} \lambda q,
$$

by (6.10) and (6.3). Hence $q$ vanishes identically: otherwise there would exist an open set on which $\lambda$ vanished identically and $q$ had no zeros. But this and (6.10) would imply that $\psi_{z z}^{\sharp}$ is linearly independent of $\psi^{\sharp}$ and $\psi_{z}^{\sharp}$, which contradicts the assumption that $f^{\sharp}$ is totally umbilic.

Remark 6.1. Let $f: M \rightarrow S^{3}$ be an immersion obtained from a Euclidean minimal surface with planar ends or a Bryant surface with smooth ends by filling in points at the ends. Then all added points are umbilic points, because away from umbilic points the second envelope $f^{\sharp}$ of the mean curvature sphere congruence of $f$ does never intersect $f$, see $(6.9)$ : since $\mathcal{Q} \equiv 0$, either $f^{\sharp}$ is a constant point $\infty$ (in the Euclidean minimal case) or $f^{\sharp}$ is the restriction of the hyperbolic Gauss map to the complement of the set of umbilic points (in the Bryant case). But in the Euclidean minimal case the planar ends of $f$ are the points where $f$ goes through $\infty$ and in the Bryant case the smooth Bryant ends of $f$ are the points at which $f$ coincides with the hyperbolic Gauss map (see the remark following Theorem 4.1).

The Willmore energy of an immersion $f: M \rightarrow S^{3}$ with $\mathcal{Q} \equiv 0$ of a compact surface into the conformal 3 -sphere is always an integer multiple of $4 \pi$ (it is essentially $4 \pi$ times the number of ends, except that smooth catenoid cousin ends have to be counted with the pole order of $F$, see Theorem 5.1). If $M=\mathbb{C P}^{1}$, the possible Willmore energies are $W=4 \pi d, d \in \mathbb{N} \backslash\{0,2,3,5,7\}$, see $[7,8]$ for the Euclidean minimal case and Theorem 3.2 for the Bryant case. 


\section{Appendix A}

The total absolute curvature plays an important role in the theory of Euclidean minimal and Bryant surfaces. In this appendix we recall its relation to the Willmore energy and show that if a Bryant surface in $\mathbf{B}^{3}$ is the intersection of $\mathbf{B}^{3}$ with a compact surface in $\mathbb{R}^{3}$ then it is a compact Bryant surface with smooth ends.

Consider an immersion $f: M \rightarrow \bar{M}$ of an oriented two-dimensional manifold $M$ into a three-dimensional Riemannian manifold $\bar{M}$. Let $d A$ be the area element, $H$ the mean curvature, $G$ the Gaussian curvature (the determinant of the Weingarten operator), $K$ the curvature of the induced metric on $M$ and $\bar{K}$ the sectional curvature of $\bar{M}$ on the tangent spaces of $f$. The latter three quantities are related by the Gauss equation $K=G+\bar{K}$. The 1 -form

$$
\left(H^{2}-G\right) d A
$$

is invariant under conformal changes of the ambient metric. If $M$ is compact of genus $g$, then the Willmore energy

$$
W=\int_{M}\left(H^{2}-G\right) d A+2 \pi \chi(M)
$$

is also conformally invariant. Note that if $\bar{K}=0$ and $M$ is compact then the Willmore energy is the $L^{2}$-norm of the mean curvature, because $2 \pi \chi(M)=$ $\int_{M} K d A=\int_{M} G d A$.

Compact Bryant surfaces with smooth ends in $\mathbf{B}^{3} \subset \mathbb{R}^{3}$ and complete minimal surface of finite total curvature with planar ends in $\mathbb{R}^{3}$ extend, adding a finite number of ends, to compact surfaces in the conformal 3-sphere $S^{3}=\mathbb{R}^{3} \cup\{\infty\}$. Their Willmore energy is related to the intrinsic absolute total curvature with respect to the corresponding space form geometry by

$$
\int_{M \backslash\{\mathrm{ends}\}}|K| d A=\int_{M}\left(H^{2}-G\right) d A=W-2 \pi \chi(M) .
$$

The first equality follows from the Gauss equation, because $H^{2}=-\bar{K}$ for both Bryant surfaces and minimal surfaces in Euclidean space.

Proposition A.1. An immersed compact surface in $\mathbb{R}^{3}$ whose non-empty intersection with $\mathbf{B}^{3}$ is a Bryant surface is a compact Bryant surface with smooth ends. In particular, except for finitely many ends the immersion is contained in $\mathbf{B}^{3}$. 
Proof. Let $f: M \rightarrow \mathbb{R}^{3}$ be a conformal immersion of a compact Riemann surface $M$ and let $M_{0}=f^{-1}\left(\mathbf{B}^{3}\right)$ such that $f_{\mid M_{0}}: M_{0} \rightarrow \mathbf{B}^{3}$ parametrizes a complete Bryant surface. The pull back to $M_{0}$ of the hyperbolic metric on $\mathbf{B}^{3}$ has non-positive curvature $K$, because $K=G+\bar{K}=-\left(H^{2}-G\right) \leq 0$, and the total absolute curvature of $f_{\mid M_{0}}$ is bounded by $\int_{M}\left(H^{2}-G\right) d A$. So $f_{\mid M_{0}}$ induces a complete metric with non-positive curvature and bounded total curvature on $M_{0}$, which implies that $M \backslash M_{0}$ is a finite number of points, cf. [16, Chapter III, Proposition 16].

\section{Acknowledgments}

We thank Ulrich Pinkall, Alexander Bobenko, Tatyana Pavlyukevich, Udo Hertrich-Jeromin and Wayne Rossmann for helpful discussions. Research for this paper was supported by DFG SPP 1154 "Global Differential Geometry".

\section{References}

[1] A.I. Bobenko, T.V. Pavlyukevich and B.A. Springborn, Hyperbolic constant mean curvature one surfaces: Spinor representation and trinoids in hypergeometric functions, Math. Z. 245(1) (2003), 63-91.

[2] C. Bohle, Möbius invariant flows of Tori in $S^{4}$, Thesis, TU-Berlin, edocs.tu-berlin.de/diss/2003/bohle_christoph.htm, 2003.

[3] C. Bohle, Constant mean curvature tori as stationary solutions to the Davey-Stewartson equation, arXiv:0810.1238 [math.DG].

[4] C. Bohle, K. Leschke, F. Pedit and U. Pinkall, Conformal maps from a 2-torus to the 4-sphere, arXiv:0712.2311v1 [math.DG]

[5] C. Bohle and G.P. Peters, Soliton spheres, arXiv:0905.2162v1 [math.DG].

[6] C. Bohle, G.P. Peters and U. Pinkall, Constrained Willmore surfaces, Calc. Var. Partial Differ. Equ. 32(2) (2008), 263-277.

[7] R.L. Bryant, A duality theorem for Willmore surfaces. J. Differ. Geom. 20(1) (1984), 23-53.

[8] R.L. Bryant, Surfaces of mean curvature one in hyperbolic space, Théorie des variétés minimales et applications (Palaiseau, 1983-1984), Astérisque No. 154-155 (1987), 12, 321-347, 353 (1988). 
[9] R.L. Bryant, Surfaces in conformal geometry, The mathematical heritage of Hermann Weyl (Durham, NC, 1987), Proc. Sympos. Pure Math., 48, Amer. Math. Soc., Providence, RI, 1988, 227-240.

[10] F.E. Burstall, D. Ferus, K. Leschke, F. Pedit and U. Pinkall, Conformal Geometry of Surfaces in $S^{4}$ and Quaternions, Lecture Notes in Mathematics, 1772, Springer, Berlin, 2002.

[11] F.E. Burstall, F. Pedit and U. Pinkall, Schwarzian derivatives and flows of surfaces, Contemp. Math. 308 (2002), 39-61.

[12] P. Collin, L. Hauswirth and H. Rosenberg, The geometry of finite topology Bryant surfaces, Ann. of Math. (2) 153(3) (2001), 623-659.

[13] D. Ferus, K. Leschke, F. Pedit and U. Pinkall, Quaternionic holomorphic geometry, Plücker formula, Dirac eigenvalue estimates and energy estimates of harmonic 2-tori, Invent. Math. 146 (2001), 507-593.

[14] U. Hertrich-Jeromin, Introduction to Möbius differential geometry. Lecture Notes Series, 300, Cambridge University Press, Cambridge, 2003.

[15] U. Hertrich-Jeromin, E. Musso and L. Nicolodi, Möbius geometry of surfaces of constant mean curvature 1 in hyperbolic space, Ann. Global Anal. Geom. 19(2) (2001), 185-205.

[16] H.B. Lawson, Jr., Lectures on minimal submanifolds. I, Mathematics Lecture Series, 9, Publish or Perish, Berkeley, 1980.

[17] F. Pedit and U. Pinkall, Quaternionic analysis on Riemann surfaces and differential geometry, Doc. Math. J. DMV, II (1998), 389-400. (Extra Volume ICM Berlin)

[18] G.P. Peters, Soliton spheres, Thesis, TU-Berlin, edocs.tu-berlin.de/diss/2004/ peters_guenter.htm, 2004.

[19] J. Richter, Conformal maps of a Riemann surface into the space of quaternions, Thesis, TU-Berlin, 1997.

[20] H. Rosenberg, Bryant surfaces, The global theory of minimal surfaces in flat spaces (Martina Franca, 1999), Lecture Notes in Math., 1775, Springer, Berlin, 2002, 67-111.

[21] W. Rossman, M. Umehara and K. Yamada, Irreducible constant mean curvature 1 surfaces in hyperbolic space with positive genus, Tohoku Math. J. (2) 49(4) (1997), 449-484. 
[22] W. Rossman, M. Umehara and K. Yamada, Mean curvature 1 surfaces in hyperbolic 3-space with low total curvature. II, Tohoku Math. J. (2) 55(3) (2003), 375-395.

[23] W. Rossman, M. Umehara and K. Yamada, Mean curvature 1 surfaces in hyperbolic 3-space with low total curvature I, Hiroshima Math. J. 34(1) (2004), 21-56.

[24] I.A. Taimanov, The Weierstrass representation of spheres in $\mathbb{R}^{3}$, the Willmore numbers, and soliton spheres, Proc. Steklov Inst. Math. 225(2) (1999), 322-343.

[25] R. Sa Earp and E. Toubiana, On the geometry of constant mean curvature one surfaces in hyperbolic space, Illinois J. Math. 45(2) (2001), 371-401.

[26] M. Umehara and K. Yamada, Complete surfaces of constant mean curvature 1 in the hyperbolic 3-space, Ann. of Math. (2) 137(3) (1993), 611-638.

InSTITUTE FÜR MATHEMATIK

TeChNische Universität BerLin

StRasse DES 17. Juni 136

10623 BERLIN

Germany

E-mail address: bohle@math.tu-berlin.de; peters@math.tu-berlin.de

RECeived January 20, 2009 
